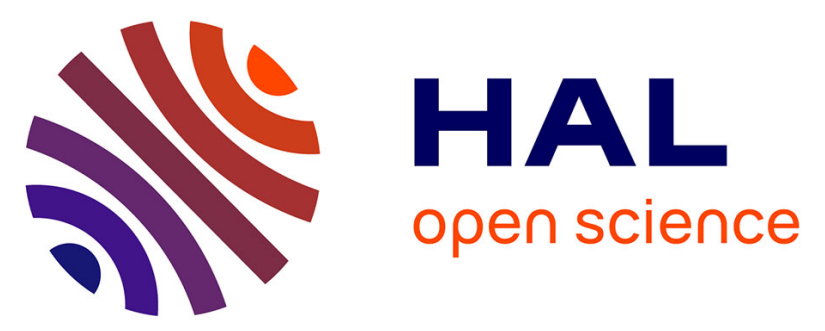

\title{
A model of heterogeneous thermoviscoplastic material preserving uniform normal strains under combined compression, tension (or compression) and shearing. Instability and homogenization results \\ Georges Chatzigeorgiou, Nicolas Charalambakis
}

\section{To cite this version:}

Georges Chatzigeorgiou, Nicolas Charalambakis. A model of heterogeneous thermoviscoplastic material preserving uniform normal strains under combined compression, tension (or compression) and shearing. Instability and homogenization results. Composites Part B: Engineering, 2019, 167, pp.267273. 10.1016/j.compositesb.2018.12.022 . hal-01963542

\author{
HAL Id: hal-01963542 \\ https://hal.science/hal-01963542
}

Submitted on 21 Dec 2018

HAL is a multi-disciplinary open access archive for the deposit and dissemination of scientific research documents, whether they are published or not. The documents may come from teaching and research institutions in France or abroad, or from public or private research centers.
L'archive ouverte pluridisciplinaire HAL, est destinée au dépôt et à la diffusion de documents scientifiques de niveau recherche, publiés ou non, émanant des établissements d'enseignement et de recherche français ou étrangers, des laboratoires publics ou privés. 


\section{Accepted Manuscript}

A model of heterogeneous thermoviscoplastic material preserving uniform normal strains under combined compression, tension (or compression) and shearing. Instability and homogenization results

George Chatzigeorgiou, Nicolas Charalambakis

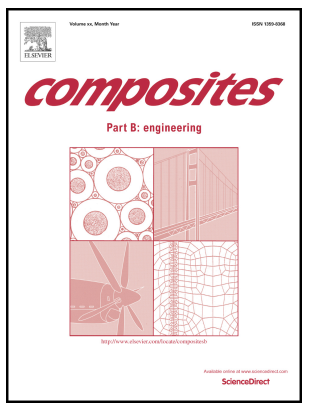

PII:

$$
\text { S1359-8368(18)32604-0 }
$$

DOI: https://doi.org/10.1016/j.compositesb.2018.12.022

Reference: JCOMB 6351

To appear in: Composites Part B

Received Date: 13 August 2018

Revised Date: 9 October 2018

Accepted Date: 6 December 2018

Please cite this article as: Chatzigeorgiou G, Charalambakis N, A model of heterogeneous thermoviscoplastic material preserving uniform normal strains under combined compression, tension (or compression) and shearing. Instability and homogenization results, Composites Part B (2019), doi: https://doi.org/10.1016/j.compositesb.2018.12.022.

This is a PDF file of an unedited manuscript that has been accepted for publication. As a service to our customers we are providing this early version of the manuscript. The manuscript will undergo copyediting, typesetting, and review of the resulting proof before it is published in its final form. Please note that during the production process errors may be discovered which could affect the content, and all legal disclaimers that apply to the journal pertain. 


\title{
A model of heterogeneous thermoviscoplastic material preserving uniform normal strains under combined compression, tension (or compression) and shearing. Instability and homogenization results
}

\author{
George Chatzigeorgiou ${ }^{\mathrm{a}}$, Nicolas Charalambakis ${ }^{\mathrm{b}}$ \\ ${ }^{a}$ LEM3-UMR 7239 CNRS, Arts et Métiers ParisTech, 4 Rue Augustin Fresnel 57078 \\ Metz, France. \\ ${ }^{b}$ Department of Civil Engineering, Aristotle University of Thessaloniki and Center for \\ Research and Development od Advanced Materials CERDAM, AUTH-Texas AM, GR \\ 54124, Thessaloniki, Greece
}

\section{Abstract}

In this paper, we present the special solution of the two-dimensional problem of a continuously graded composite made of thermovisco- rigid plastic materials under combined biaxial quasistatic compression and tension (or compression) and shear, that preserves prescribed uniform normal strains. The related reference initial-boundary value problem is fully defined and the corresponding solution is analyzed and computed numerically. In the context of a linearized instability analysis, critical conditions, as the critical shear banding angle, in terms of the loading level and material heterogeneities, are presented. In the context of non-linear instability, these results are examined and explained. Additionally, in the same context of non-linear analysis, the destabilizing mechanism, the onset of instability and the critical time for prescribed lower and upper bounds of equivalent strain-rate and upper bound of equivalent strain are defined and related to the lateral normal stress. The limitations of linearized analysis results are also revealed. Moreover, 
a semi-analytical homogenization scheme for a periodically-graded plate is presented. The related results are used for the derivation of a homogenized problem of a multilayered composite with continuously graded interlayers. Keywords: Thermo-visco-plasticity; Instability; Continuously graded structures; Homogenized problem.

\section{Introduction}

Modeling the mechanical behavior of heterogeneous thermoviscoplastic materials has been the subject of many research papers from both mathematical and computational point of view (Batra and Love, 2004; Charalambakis and Baxevanis, 2004; Batra and Love, 2005; Terada and Kikuchi, 2001; Chatzigeorgiou and Charalambakis, 2005; Chaboche et al., 2005; Love and Batra, 2006; Chatzigeorgiou et al., 2007, 2009; Cavalcante et al., 2009; Pindera et al., 2009; Cavalcante et al., 2009; Khatam and Pindera, 2010; Nie and Batra, 2010; Nie et al., 2011; Nackenhorst et al., 2011, Charalambakis et al., 2018). We also refer to Michopoulos et al., 2018 for a presentation of the enabling technology for collecting data that summarize the dissipation behavior of composites under quasistatic multiaxial loading, via robotic testing. The one-dimensional homogeneous case has been completely investigated by Bai, 1982; Anand et al., 1987; Fressengeas and Molinari, 1987 and the related instability criteria for shear banding have been introduced. The biaxial loading of viscoplastic and thermoviscoplastic homogeneous materials has been presented in Batra and Liu, 1990; Dudzinski and Molinari, 1991. The biaxial loading of functionally graded multi-metallic composites bas been studied in Batra and Love, 2004, 2005. The compressive or shear failure of composites 
has been the subject of many works, as Basu et al., 2006; Edgren et al., 2006; Bai and Keller, 2009; Khedmati et al., 2014. Unexpected phenomena, as in multilayered copper subjected to plane strain compression, were experimentally observed, revealing different kinds of interface instability (saw-thoothed, wavy, double undulation, bifurcation, see the interesting paper Gotoh et al., 1993). Experimental performances and modeling of old structures with steel bearings (see Mander et al., 1993), as in problems of quasi-static fragility of multi-span bridges with drop spans, need also insights on the behavior of metallic multilayers. Of special interest are the studies on the mechanism of shear banding related to the interfacial weakening leading to strain softening in nanolayered composites (Mara et al., 2010): geometric stress concentrations exert a non-uniaxial stress state, causing rotation of the layers thus their shear loading. Intense research effort has been devoted recently to the emergence of stable interfaces under extreme plastic deformation (Beyerlein et al., 2014), to the cyclic indentation behavior of metal-ceramic nanolayered composites (Shen et al., 2012) and to the high-strength and thermally stable bulk nanolayered composites due to twin-induced interfaces ( (Zheng et al., 2013). Interfaces can be designed within bulk nanostructured composites to enhance previously manufactured bulk nanocrystalline materials with respect to mechanical and thermal performance, Shen et al., 2012; Zheng et al., 2013, see also Deng et al., 2005 for metal-ceramic and Ovid'ko, 2014 for metal-graphene composites. Very recently, thermo-hydro-mechanical couplings and strain localization in $3 D$ Cosserat continua are presented in two companion papers (Rattez et al., 2018a, Rattez et al., 2018b).

In this paper we present the loading conditions and the corresponding so- 
lution for a special uniform normal strain-rate biaxial deformation of a plate made of heterogeneous rigid thermoviscoplastic von Mises material with continuously graded properties following one direction. This structure could be the idealization of a composite formed by alternate layers and functionally graded interfaces. Compressive or shear failure is related to material instabilities as shear banding, enhanced by the heterogeneities of thermomechanical and other physical parameters. Additionally, interfacial weakening and/or interfacial stress concentrations may act as precursors of instabilities. It is shown that, if it is assumed a uniform normal stress distribution in the direction vertical to the material gradation, then the special solution of the above problem requires a continuously varying lateral normal stress distribution in the direction of gradation. It is shown that this situation yields to shear stress, shear strain and temperature distributions, also dependent on the direction of gradation. This solution first is considered as a non-uniform ground solution for the detailed instability analysis of the above heterogeneous problem. As expected, the linearized instability analysis reveals the interplay between thermomechanical heterogeneities and actual strain and stress level on the emergence of fast growing perturbations, as well as their direction. The principal aims of the present work is, first to apply non-linear analysis techniques in order to explain the instability criterion given by the linearized analysis and reveal the onset and evolution of stable and unstable processes in real time, and second to describe quantitatively the thermomechanical process in real time. We note that the above solution excludes behavior under cyclic loading (for cyclic loading resulting to accelerate cyclic softening, we refer to Benaarbia et al., 2018). 
Moreover, the above solution gives, for the special case of a very fine periodic gradation of material properties under stability conditions, a homogenization result that defines the loading conditions (combined biaxial compression and shearing or combined compression-tension and shearing) of the heterogeneous case, preserving uniform normal straining in both directions. Today's homogenization methods of elastic or inelastic materials are necessarily based on computational methods (for a very recent application of the Finite-Volume Direct Averaging Micromechanics (FVDAM) to simulate the multiphase heterogeneous materials in three-dimensional domain we refer to Chen et al., 2018a), with very often computer-aided generation of $3 D$ representative volume elements (see the very interesting paper by Bargmann et al., 2018). Indeed, only one-dimensional inelastic problems can be investigated semi-analytically. In the present paper, a semi-analytical method is applied in order to solve the $2 D$ non-linear homogenization problem of biaxial loading of multilayers. For two different points of view on homogenization of non-linear laminated composites we refer to Omri et al., 2000, LopezRealpozo et al., 2008, Zhu et al., 2018. For the analysis of effective behavior of elastic structures with non-linear periodicity we refer to Guinovart-Sanjuan et al., 2018 and Dong et al., 2018. For an application of the finite-volume homogenization to nanoporous materials with cylindrical voids we refer to Chen et al., 2018b.

The homogenization result of the present paper can eventually be used in manufacturing processes of layered materials with functionally graded interfaces, for which prescribed uniform normal strains are desired under combined biaxial normal stress and shearing. More specifically, the lateral effec- 
tive tension can be adjusted in order that given uniform normal strain-rate is preserved, resulting to uniform elongation or shortening of components, since eventual strain non-uniformities, due to elastic deformation, are negligible. On the other hand, since the heterogeneous lateral stress is completely defined, high stress concentrations due to material non-uniformities can be controlled at every moment. Additionally, in case of compressive lateral stress, plastic microbuckling issues can eventually be considered (see Jelf and Fleck, 1994). Moreover, from the heterogeneous shear strain distribution, high non-uniformities can also be examined.

\section{Statement of the problem}

We consider a heterogeneous plate made of thermoviscoplastic materials with continuous or moderate discontinuous properties varying with respect to the $y$-axis (Figure 1). This case could correspond to a functionally graded material or to a multilayered material with layers parallel to the $x$-axis, in which interfaces are approximated by continuously graded layers exhibiting smooth properties with respect to $y$. We assume that the body is deformed under plane strain conditions in the plane $O x y$ with $\varepsilon_{z z}=0$. The deformation process is described by: (i) The momentum equations

$$
\begin{aligned}
& \varrho \frac{\partial v_{x}}{\partial t}=\frac{\partial \sigma_{x x}}{\partial x}+\frac{\partial \sigma_{x y}}{\partial y}, \\
& \varrho \frac{\partial v_{y}}{\partial t}=\frac{\partial \sigma_{y x}}{\partial x}+\frac{\partial \sigma_{y y}}{\partial y},
\end{aligned}
$$

where $v_{x}, v_{y}$ are the velocity components and $\sigma_{x x}, \sigma_{x y}, \sigma_{y y}$ are the stress components. (ii) The energy equation (repetition of indices $i, j$ means summation 
over $\mathrm{x}, \mathrm{y}, \mathrm{z})$

$$
c \frac{\partial \theta}{\partial t}=k \frac{\partial^{2} \theta}{\partial x^{2}}-\theta C_{i j k l} \dot{\varepsilon}_{k l}^{e} \alpha_{i j}+\sigma_{i j} \dot{\varepsilon}_{i j}^{p}-\frac{\partial F(\theta, p)}{\partial p} \dot{p}+\frac{\partial^{2} F(\theta, p)}{\partial \theta \partial p} \theta \dot{p},
$$

where $\theta$ is the temperature field, $p$ the accumulated strain and $F(\theta, p)$ a hardening function coming from the free energy function. (iii) The plastic flow equations corresponding to the von Mises criterion

$$
\dot{\varepsilon}_{x x}^{p}=D_{x} \dot{p}, \quad \dot{\varepsilon}_{y y}^{p}=D_{y} \dot{p}, \quad \dot{\varepsilon}_{x y}^{p}=D_{x y} \dot{p}
$$

(iv) The compatibility equation

$$
\frac{\partial^{2} \dot{\varepsilon}_{x x}}{\partial y^{2}}+\frac{\partial^{2} \dot{\varepsilon}_{y y}}{\partial x^{2}}=2 \frac{\partial^{2} \dot{\varepsilon}_{x y}}{\partial x \partial y}
$$

where $\varepsilon_{i j}=\varepsilon_{i j}^{e}+\varepsilon_{i j}^{p}$ is the (small) strain decomposed into elastic and plastic part, while

$$
p(t)=p\left(t_{0}\right)+\int_{t_{0}}^{t} \dot{p}(\tau) d \tau
$$

stands for the accumulated strain and the dot denotes the time partial derivative. We note that, from $(2.4), \dot{p}=\sqrt{\frac{4}{3}\left(\left(\dot{\varepsilon}_{x x}^{p}\right)^{2}+\left(\dot{\varepsilon}_{x y}^{p}\right)^{2}\right)}$. The plastic strain is assumed to be incompressible, and since $\varepsilon_{z z}=0$,

$$
\varepsilon_{x x}=-\varepsilon_{y y}
$$

In (2.3) the first two terms in the right hand side are due to thermoelastic effects, while the rest three terms are due to inelastic effects. In the von Mises criterion $\bar{\sigma}-f(\theta, p, \dot{p}) \leq 0$, the equivalent stress $\bar{\sigma}$ is given by

$$
\bar{\sigma}=\sqrt{\frac{3}{4}\left(\sigma_{x x}-\sigma_{y y}\right)^{2}+3 \sigma_{x y}^{2}}
$$

$\operatorname{In}(2.4)$

$$
D_{x}=-D_{y}=\frac{3}{4 \bar{\sigma}}\left(\sigma_{x x}-\sigma_{y y}\right), \quad D_{x y}=\frac{3}{2 \bar{\sigma}} \sigma_{x y}
$$


The process starts at a time, which is assumed to coincide with the time at which all material regions deform plastically with negligible elastic effects (including initial elastic strain-nonuniformities), providing the main process with initial, nearly uniform, effective strains $p_{0}(y)$ and causing strains $\varepsilon_{i j} \equiv$ $\varepsilon_{i j}^{p}$. Therefore, for $t>0$ the energy equation involves only inelastic effects and under adiabatic conditions is expressed as

$$
\frac{\partial \theta}{\partial t}=c^{*} \sigma_{i j} \dot{\varepsilon}_{i j}
$$

which, for a von Mises material with $\dot{\varepsilon}_{i j}^{p}=\frac{3}{2 \bar{\sigma}} s_{i j}$, is equivalent to

$$
\frac{\partial \theta}{\partial t}=c^{*} \bar{\sigma} \dot{p}
$$

The constitutive law is assumed of the form

$$
\bar{\sigma}=G \theta^{\alpha} p^{n} \dot{p}^{m} .
$$

The material parameters $\varrho$ (density), $c$ (specific heat), $c^{*}=\frac{\beta}{\varrho c}$, (where $\beta$ is the Taylor-Quinney coefficient) and $G$, are all assumed to depend only on $y$. As mentioned, they are approximated by smooth functions of $y$.

Using non-linear analysis techniques in order to study the qualitative behavior of the solution of the above problem described by (2.1), (2.2), (2.11), (2.4), (2.5), (2.6), (2.7), (2.8), (2.9), (2.12) under appropriate initial and boundary conditions for velocity and/or stress, leads to the conclusion that, if for some material point, the (heterogeneous) thermal softening is sufficiently large in order to overcome the stabilizing effect of strain-hardening and strain-rate sensitivity, then, after a strain and loading level, for this point there exists a critical time at which the equivalent strain-rate increases without bound (see Appendix A. See also for the one-dimensional homogeneous 
case Tzavaras, 1987, Katsaounis et al., 2017 and for the non-homogeneous case under plane stress conditions Chatzigeorgiou and Charalambakis, 2005; Chatzigeorgiou et al., 2007). But this finding is without practical interest since it corresponds to an infinite value of strain rate, while problems we are interested in involve not only finite strain-rates, but of bounded measures, as in quasistatic processes. Therefore, a realistic approach is to consider a finite critical strain rate and to compute the critical time corresponding to that value from (9.2) (see Appendix A). Consequently, questions about time-asymptotic stability of the solutions of the above problem are out of the scope of the present paper.

\section{The quasistatic case}

In order to study in detail the onset, geometry and evolution of instability for the above problem we consider its quasi-static approximation under appropriate initial and boundary conditions for velocity and/or stress. In Subsection 3.1 we find a special solution for the system of: the equilibrium equations

$$
\begin{aligned}
& \frac{\partial \sigma_{x x}}{\partial x}+\frac{\partial \sigma_{x y}}{\partial y}=0 \\
& \frac{\partial \sigma_{y x}}{\partial x}+\frac{\partial \sigma_{y y}}{\partial y}=0
\end{aligned}
$$

the energy equation (2.11), the plastic flow rule (2.4), the compatibility condition (2.5), the accumulated plastic strain (2.6), the incompressibility condition (2.7), the von Mises stress (2.8), the relation between the flow coefficients and the stress (2.9) and the constitutive law (2.12). This solution will first serve in Subsection 3.2 for the linearized instability analysis. We will consider it as a "ground" solution $S^{0}$ for the above heterogeneous problem and 
we will superpose a perturbation of the form $\delta S=\delta S^{0} e^{\eta \Delta t} e^{i \xi x_{1}}$ following a $x_{1}$-direction, causing a possible instability in $x_{2}$-direction.

The instability or stability depends on the sign (positive or negative, respectively) of the parameter $\eta$. This will be deduced from the solution of the determinant of the linear homogeneous system resulting after substitution of

$$
S=S^{0}+\delta S
$$

into momentum equations (3.1), (3.2), energy equation (2.11), plastic flow equation (2.4), compatibility equation (2.5), accumulated strain (2.6), incompressibility equation (2.7), equivalent stress (2.8), plastic flow coefficients (2.9) and constitutive equation (2.12), written with respect to the $O x_{1} x_{2^{-}}$ system. The stress level $\sigma_{11}^{0}, \sigma_{22}^{0}, \sigma_{12}^{0}$ is expressed in terms of the angle $\psi$ between axes $O x_{1}$ and $O x$ of the orthogonal systems $O x y$ and $O x_{1} x_{2}$ and the components $\sigma_{x x}^{0}, \sigma_{y y}^{0}, \sigma_{x y}^{0}$ (see Appendix A).

The plastic flow and the compatibility equation are written as

$$
\begin{gathered}
\dot{\varepsilon}_{11}=D_{1} \dot{p}, \quad \dot{\varepsilon}_{22}=D_{2} \dot{p}, \quad \dot{\varepsilon}_{12}=D_{12} \dot{p} \\
\frac{\partial^{2} \dot{\varepsilon}_{11}}{\partial x_{2}^{2}}+\frac{\partial^{2} \dot{\varepsilon}_{22}}{\partial x_{1}^{2}}=2 \frac{\partial^{2} \dot{\varepsilon}_{12}}{\partial x_{1} \partial x_{2}}
\end{gathered}
$$

where

$$
D_{1}=-D_{2}=\frac{3}{4 \bar{\sigma}}\left(\sigma_{11}-\sigma_{22}\right), \quad D_{12}=\frac{3}{2 \bar{\sigma}} \sigma_{12}
$$

and

$$
\bar{\sigma}=\sqrt{\frac{3}{4}\left(\sigma_{11}-\sigma_{22}\right)^{2}+3 \sigma_{12}^{2}} .
$$

Equation of energy and constitutive law are written as (2.11) and (2.12).

In the next Section 4, this special solution will serve for the non-linear instability analysis. 


\subsection{The reference solution}

In this subsection, we will define a special solution $S^{0}$ for the quasistatic case under the boundary conditions shown in Figure 1, consisting of given normal strain rates

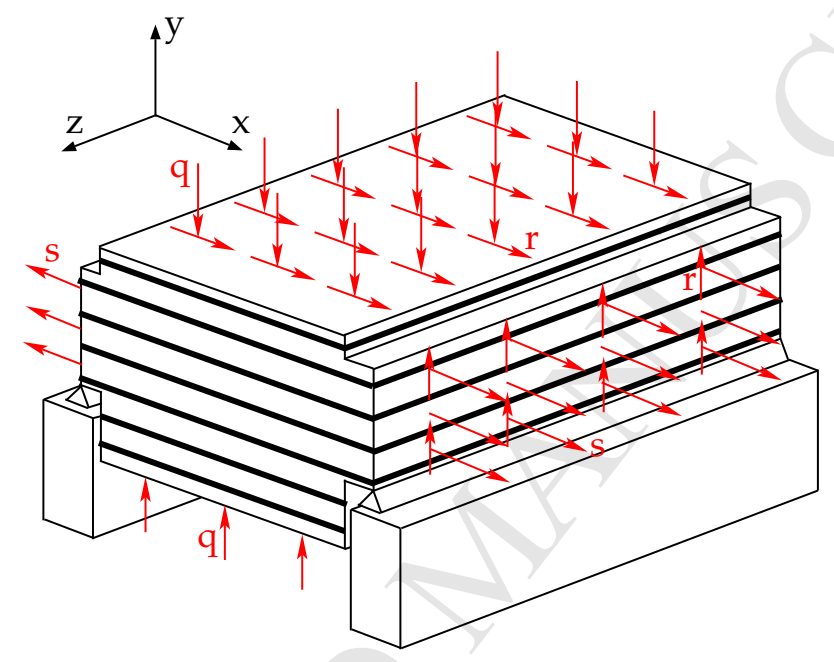

(a)

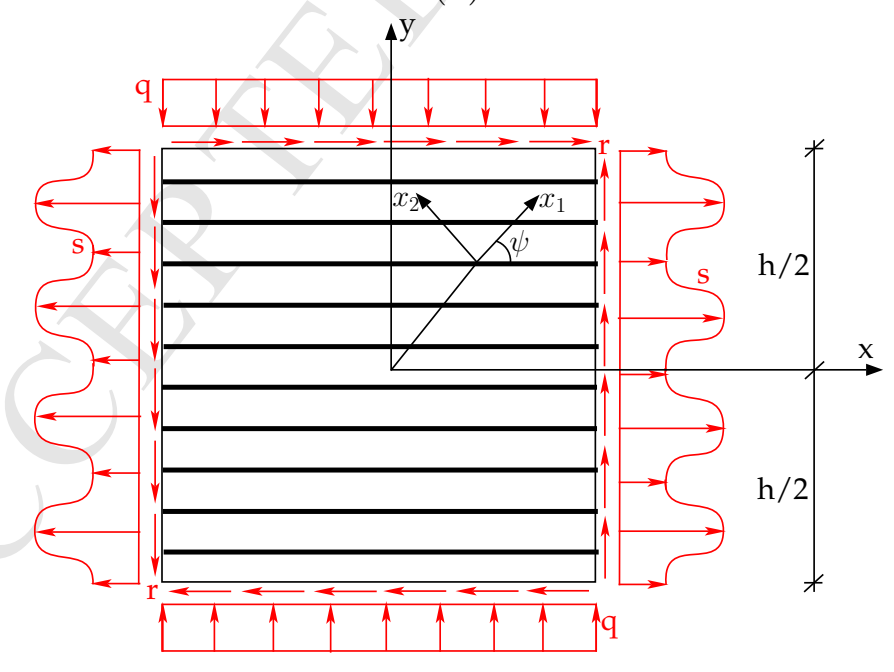

(b)

Figure 1: Composite structure and loading conditions 


$$
\dot{\varepsilon}_{x x}^{0}=-\dot{\varepsilon}_{y y}^{0}=v,
$$

which correspond to a velocity field

$$
v_{x}^{0}=v x+\phi(y, t), \quad v_{y}^{0}=-v y,
$$

and of stress components

$$
\sigma_{x x}^{0}=s(y, t) \quad \sigma_{y y}^{0}=-q(t), \quad \sigma_{x y}^{0}=r(t)
$$

We assume that we control the process at every time step in order that the unknown shear strain remains quasi-static,

$$
\dot{\varepsilon}_{x y}=\frac{1}{2} \frac{\partial \varphi}{\partial y} \leq 10^{-2}
$$

This needs a sufficiently small given normal strain-rate, for instance $v \sim 10^{-3}$. We easily verify that the solution $S^{0}=\left(\sigma_{x x}^{0}, \sigma_{y y}^{0}, \sigma_{x y}^{0}, \bar{\sigma}^{0}, \dot{\varepsilon}_{x x}^{0}, \dot{\varepsilon}_{y y}^{0}, \dot{\varepsilon}_{x y}^{0}, \dot{p}^{0}\right.$, $\theta^{0}$ ) satisfies momentum (3.1), (3.2), compatibility (2.5) and incompressibility equations (2.7). Given the functions $v, q(t), r(t)$, we can define the unknown functions $s(y, t)$ and $\theta^{0}(y, t)$ from plastic flow (2.4), accumulated strain (2.6), equivalent stress (2.8), constitutive law (2.12) and energy equation (2.11).

We also find that

$$
\begin{gathered}
\bar{\sigma}^{0}(y, t)=\sqrt{\frac{3}{4}(s(y, t)+q(t))^{2}+3 r(t)^{2}}, \\
\dot{p}^{0}=\frac{4}{3} \frac{v \bar{\sigma}^{0}(y, t)}{s(y, t)+q(t)}, \\
\dot{\varepsilon}_{x y}^{0}=\frac{2 v r(t)}{s(y, t)+q(t)} .
\end{gathered}
$$


Then $s(y, t), \theta^{0}(y, t)$ are defined from the solution of the system of the equivalent stress (3.12), equivalent strain-rate (3.13), constitutive law (2.12), accumulated plastic strain (2.6) and energy equation (2.11).

It is worth noticing that the process is thermodynamically consistent if and only if

$$
s(y, t)+q(t)>0, \quad \dot{p}>0 .
$$

However, more restrictive conditions on $s, \dot{p}$ are needed. These conditions are due to the restriction that the process is quasistatic, imposing an upper bound on the equivalent strain-rate

$$
\dot{p}<10^{-2}
$$

From $\dot{\varepsilon}_{x y}^{2}=\frac{3}{4} \dot{p}^{2}\left(1-\frac{4}{3}\left(\frac{v}{\tilde{p}}\right)^{2}\right)$, shear strain rate (3.14) and equivalent strain rate (3.13) we obtain

$$
\begin{gathered}
\dot{\varepsilon}_{x y}=\frac{\sqrt{3}}{2} \dot{p} \sqrt{1-\frac{4}{3} \frac{v^{2}}{\dot{p}^{2}}} \operatorname{sign}(r), \\
s+q=\frac{|r|}{\sqrt{\frac{3}{16} \frac{\dot{p}^{2}}{v^{2}}-\frac{1}{4}}},
\end{gathered}
$$

while from $\frac{r}{\dot{\varepsilon}_{x y}}=\frac{2}{3} \overline{\frac{\sigma}{p}}$ we obtain

$$
\bar{\sigma}=\frac{\sqrt{3}|r|}{\sqrt{1-\frac{4}{3} \frac{v^{2}}{\dot{p}^{2}}}} .
$$

Then, from the bound of strain-rate (3.16) and (3.18),

$$
s>0.233 r-q,
$$

while from $1-\frac{4}{3} \frac{v^{2}}{\dot{p}^{2}}>0$

$$
\dot{p}>1.155 v \text {. }
$$


Note 1: The special case $v=0$ corresponds to $s(y, t)=-q$ and leads to pure shearing with $v_{x}=\varphi(y, t), v_{y}=0, \dot{\varepsilon}_{x y}=\frac{1}{2} \frac{\partial \varphi}{\partial y}, \dot{p}=\frac{1}{\sqrt{3}} \frac{\partial \varphi}{\partial y}, \bar{\sigma}=\sqrt{3} r$.

It is also worth noticing that, due to the presence of $\varphi(y, t)$, the left hand side of the second equilibrium equation (3.2) is only approximately equal to zero, so the process must start and be continued by controlling the quasilinearity with respect to time of shear strain $\varepsilon_{x y}^{0}$ and accumulated strain $p^{0}(y, t)$, as in Dudzinski and Molinari, 1991; Chatzigeorgiou and Charalambakis, 2005. Let us clarify this task. Using equivalent stress (3.12) and equivalent strain (3.13) we first write all equations of the problem (equivalent strain rate (3.13), constitutive law (2.12), accumulated strain (2.6), energy (2.11)) in terms of lateral stress, temperature, equivalent strain rate and equivalent strain, $s(y, t), \theta^{0}(y, t), \dot{p}^{0}(y, t), p^{0}(y, t)$, as

$$
\begin{gathered}
\frac{3}{4} \frac{s+q}{v}=G\left(\theta^{0}\right)^{\alpha}\left(p^{0}\right)^{n}\left(\dot{p}^{0}\right)^{m-1}, \\
\frac{\partial \theta^{0}}{\partial t}=c^{*} v\left[(s+q)+4 \frac{r^{2}}{(s+q)}\right], \\
\dot{p}^{0}=v \sqrt{\frac{4}{3}\left[1+4 \frac{r^{2}}{(s+q)^{2}}\right],} \\
p^{0}(y, t)=p(0, y)+\int_{0}^{t} \dot{p}^{0}(\tau, y) d \tau .
\end{gathered}
$$

Combining the energy equation (3.23) with (3.24) gives after integration

$$
\theta^{0}(y, t)=\theta^{0}(y, 0)+\frac{4}{3} c^{*} v \int_{0}^{t}\left[\frac{3}{4}(s(y, \tau)+q)+3 r^{2} \frac{1}{(s(y, \tau)+q)}\right] d \tau,
$$

while from (3.24) and (3.25)

$$
p^{0}(y, t)=p^{0}(y, 0)+\int_{0}^{t} v \sqrt{\frac{4}{3}\left[1+4 \frac{r^{2}}{(s(y, \tau)+q)^{2}}\right]} d \tau .
$$


Then, $s$ is obtained by inserting $\theta, p^{0}$ from (3.26), (3.27) into (3.22) by the numerical solution of the integral equation

$$
H(s)=g(s)-\theta^{\alpha}(s) p(s)^{n}=0
$$

where

$$
\begin{gathered}
g(s)=c_{1}(s+q)\left(1+4 \frac{r^{2}}{(s+q)^{2}}\right)^{\frac{1-m}{2}}, \quad c_{1}=\left(\frac{3}{4}\right)^{\frac{1+m}{2}} v^{-m} G^{-1}, \\
\theta(s)=\theta_{0}+c_{2} \int_{0}^{t} h_{\theta}(s) d \tau, \quad c_{2}=c^{*} v \\
p(s)=p_{0}+c_{3} \int_{0}^{t} h_{p}(s) d \tau, \quad c_{3}=\frac{2}{\sqrt{3}} v \\
h_{\theta}(s)=s+q+4 r^{2} \frac{1}{s+q} \\
h_{p}(s)=\sqrt{1+4 \frac{r^{2}}{(s+q)^{2}}} .
\end{gathered}
$$

with initial condition

$$
\begin{aligned}
& {[s(y, 0)+q]\left[1+4 \frac{r^{2}}{(s(y, 0)+q)^{2}}\right]^{\frac{(1-m(y))}{2}}=} \\
& {\left[\frac{4}{3}\right]^{\frac{(1+m(y))}{2}} v^{m(y)} G(y) \theta^{0}(y, 0)^{\alpha(y)} p^{0}(y, 0)^{n(y)} .}
\end{aligned}
$$

From the representation of $s$ we conclude that it always depends on the time, even for steady data. Therefore, from the new expression for the equivalent strain rate $(3.24)$ it follows that $\dot{p}^{0}$ is always a function of time, even if the normal strain rates $v$ and the given shear stress $r$ are steady. That is why $\dot{p}^{0}$ must be controlled at every time step if it fits between lower and upper bounds (3.21) and (3.16). Moreover, we conclude that, if all initial conditions (temperature and strain) and thermo-mechanical parameters are periodic with respect to $y$, then $s(y, t)$ is also periodic with respect to $y$. 


\subsection{Four special cases of (3.28)}

In this subsection, we present four special cases, corresponding to (i) $\alpha=n=m=0$, (ii) $\alpha<0, n=m=0$, (iii) $n \neq 0, \alpha=m=0$, (iv) $\alpha=n=0, m \neq 0$. From the integral equation (3.28) and the new expressions for equivalent strain rate (3.24) and temperature (3.26) we obtain

(i) $\alpha=n=m=0$.

$$
\begin{aligned}
\dot{p}^{0}(y) & =\frac{4}{3} v \frac{G(y)}{\sqrt{\frac{4}{3} G^{2}(y)-4 r^{2}}}, \\
\theta^{0}(y)=c^{*} G(y) p^{0}(y), \quad s(y) & =\sqrt{\frac{4}{3} G(y)^{2}-4 r^{2}-q .}
\end{aligned}
$$

Equation (3.28) is identity.

(ii) $\alpha<0, n=m=0$ (net softening).

$$
\begin{aligned}
\dot{p}^{0}(y, t) & =\frac{4}{3} v \frac{G(y) \theta^{0}(y, t)^{\alpha(y)}}{\sqrt{\frac{4}{3} G^{2}(y) \theta^{0}(y, t)^{2 \alpha(y)}-4 r^{2}}}, \\
s(y, t) & =\sqrt{\frac{4}{3} G(y)^{2} \theta^{0}(y, t)^{2 \alpha(y)}-4 r^{2}}-q .
\end{aligned}
$$

This case is a pathological one, since energy equation reads

$$
\frac{\partial \theta^{0}(y, t)}{\partial t}=C^{*} v\left[\sqrt{\frac{4}{3} G(y)^{2} \theta^{0}(y, t)^{2 \alpha(y)}-4 r^{2}}+4 \frac{r^{2}}{\sqrt{\frac{4}{3} G(y)^{2} \theta^{0}(y, t)^{2 \alpha(y)}-4 r^{2}}}\right]
$$

and, as $\theta^{0}(., t)$ is increasing and $\alpha<0$, there exists a finite time $\bar{t}$ and a $\tilde{y}$ at which the second term increases without bound with $\theta(\tilde{y}, \bar{t})=\left[\frac{1}{\sqrt{3}} \frac{G(\tilde{y}, \bar{t})}{r}\right]^{\frac{1}{|\alpha(\tilde{y})|}}$, where $\tilde{y}$ the point of maximum temperature. 
(iii) $\alpha=m=0, n>0$ (net strain-hardening).

$$
\begin{gathered}
s(y, t)=\sqrt{\frac{4}{3} G(y)^{2} p^{0}(y, t)^{2 n(y)}-4 r^{2}}-q, \\
\frac{\partial p^{0}(y, t)}{\partial t}=\sqrt{\frac{4}{3}} v \frac{p^{0}(y, t)^{n(y)}}{\sqrt{p^{0}(y, t)^{2 n(y)}-\frac{3 r^{2}}{G(y)^{2}}}} .
\end{gathered}
$$

The solution of this case is always bounded, since

$$
\begin{array}{r}
\frac{\partial p^{0}(y, t)}{\partial t} \leq \\
\sqrt{\frac{4}{3}} v \frac{1}{\sqrt{p^{0}(\bar{y}, 0)^{2 n(\bar{y})}-\frac{3 r^{2}}{G(\bar{y})^{2}}}} p^{0}(\bar{y}, t)^{n(\bar{y})}
\end{array}
$$

where $\bar{y}$ the point of the minimum initial effective strain. It can be obtained by integration: $\int \frac{\sqrt{p^{0}(y, .)^{2 n(y)}-\frac{3 r^{2}}{G^{2}}}}{p^{0}(y,)^{n(y)}} d p^{0}(y,)=.\frac{2}{\sqrt{3}} v t$.

(iii) $\alpha=n=0, m>0$ (net strain-rate-hardening).

$$
s(y)=\sqrt{\frac{4}{3} G(y)^{2} \dot{p}^{0}(y)^{2 m(y)}-4 r^{2}}-q .
$$

In this case, $\dot{p}^{0}, s(y)$, are obtained from the algebraic equations

$$
\begin{gathered}
{\left[\sqrt{\frac{4}{3} G(y)^{2} \dot{p}^{0}(y)^{2 m(y)}-4 r^{2}}\right]\left[1+4 \frac{r^{2}}{\frac{4}{3} G(y)^{2} \dot{p}^{0}(y)^{2 m(y)}-4 r^{2}}\right]^{\frac{(1-m(y))}{2}}} \\
=\left[\frac{4}{3}\right]^{\frac{(1+m(y))}{2}} v^{m(y)} G(y) \\
s(y)+q=\left[\frac{4}{3}\right]^{\frac{m+1}{2}} v^{m(y)-1} G(y)\left[1+4\left(\frac{r}{s(y)+q}\right)^{2}\right]^{\frac{m(y)-1}{2}} .
\end{gathered}
$$

\subsection{Linearized instability analysis}

On the above defined reference solution we superpose a perturbation $\delta S=$ $\delta S^{0} e^{\eta \Delta t} e^{i \xi x_{1}}$, where $\delta S^{0}=\left(\delta \sigma_{11}^{0}, \delta \sigma_{22}^{0}, \delta \sigma_{12}^{0}, \delta \sigma^{0}, \delta \dot{\varepsilon}_{11}^{0}, \delta \dot{\varepsilon}_{22}^{0}, \delta \dot{\varepsilon}_{12}^{0}, \delta \dot{p}^{0}, \delta \theta^{0}\right)$. In Appendix B we can see the determinant of the linear homogeneous system 
which must be zero, resulting to a first order equation (omitting the equilibrium solution $X=0$ ) with solution

$$
X=\frac{\frac{c *|\alpha|}{\theta^{0}}\left[\frac{3}{4}-2\left(D_{1}^{0}\right)^{2}\right]-\frac{n}{p^{0} \bar{\sigma}^{0}}\left[\frac{3}{4}-\left(D_{1}^{0}\right)^{2}\right]}{\frac{1}{\bar{\sigma}^{0}}\left(D_{1}^{0}\right)^{2}+\frac{m}{\bar{\sigma}^{0}}\left[\frac{3}{4}-\left(D_{1}^{0}\right)^{2}\right]},
$$

where

$$
D_{1}^{0}=\frac{3}{4 \bar{\sigma}^{0}}\left(\sigma_{11}^{0}-\sigma_{22}^{0}\right)=\frac{3}{4 \bar{\sigma}^{0}}[(s(y, t)+q(t)) \cos 2 \psi+2 r(t) \sin 2 \psi],
$$

and $\bar{\sigma}^{0}$ is given by (3.12).

For instability $(X>0)$ (see Figure 2$)$ there are two conditions: the first is related to the orientation $\psi$ of $x_{1}$,

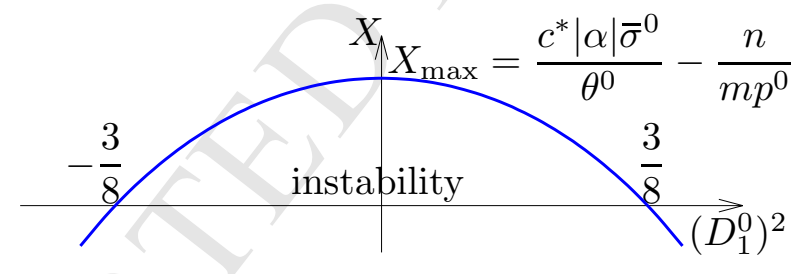

Figure 2: Relative perturbation parameter $X$ versus $\left(D_{1}^{0}\right)^{2}$ (relation (3.42))

$$
\left(D_{1}^{0}\right)^{2}<\frac{3}{8}
$$

and the second is related to the interplay between thermomechanical and loading parameters (see Figure 3), 


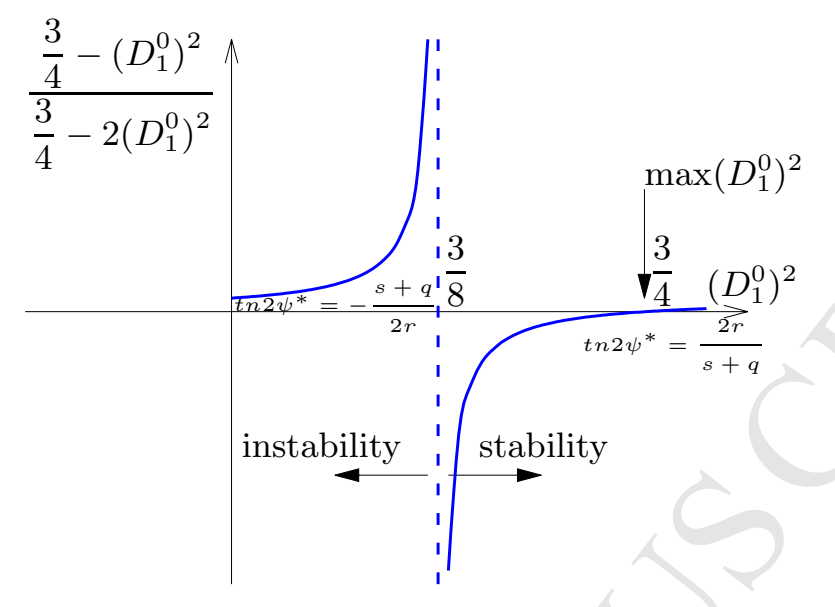

Figure 3: Interplay between thermomechanical and loading parameters (relation (3.45))

$$
\frac{\frac{c *|\alpha|}{\theta^{0}}}{\frac{n}{p^{0} \bar{\sigma}^{0}}}>\frac{\frac{3}{4}-\left(D_{1}^{0}\right)^{2}}{\frac{3}{4}-2\left(D_{1}^{0}\right)^{2}}
$$

In Figure 4 we see the regions of orientation angles susceptible to give instabilities, for given loading $r, q$ and corresponding $s$.

Next, we compute the critical angle $\psi^{*}$ by putting $\frac{\partial X}{\partial \psi}=0$. We easily verify that $\frac{\partial X(\psi)}{\partial \psi}$ and $\left(D_{1}^{0}\right)^{2}(\psi)$ become both zero for the value $\psi^{*}$ given by

$$
\operatorname{tn} 2 \psi^{*}=-\frac{s(y, t)+q(t)}{2 r(t)} .
$$

From Appendix B we deduce that, since $\left(D_{1}^{0}\right)^{2}+\left(D_{3}^{0}\right)^{2}=\frac{3}{4},\left|D_{3}^{0}\right|$ becomes maximum at $\psi=\psi^{*}$ and from the expression of $D_{3}^{0}$ we see that the shear stress following the critical angle becomes maximum too,

$$
\left|\sigma_{12}^{0}\right|=\frac{2}{3} \bar{\sigma}^{0}\left|D_{3}^{0}\right|=\frac{1}{\sqrt{3}} \bar{\sigma}^{0}
$$

forming a shear band. Weaker material zones is observed that, under nonuniaxial stress state, tend to be brought into an orientation where they are 


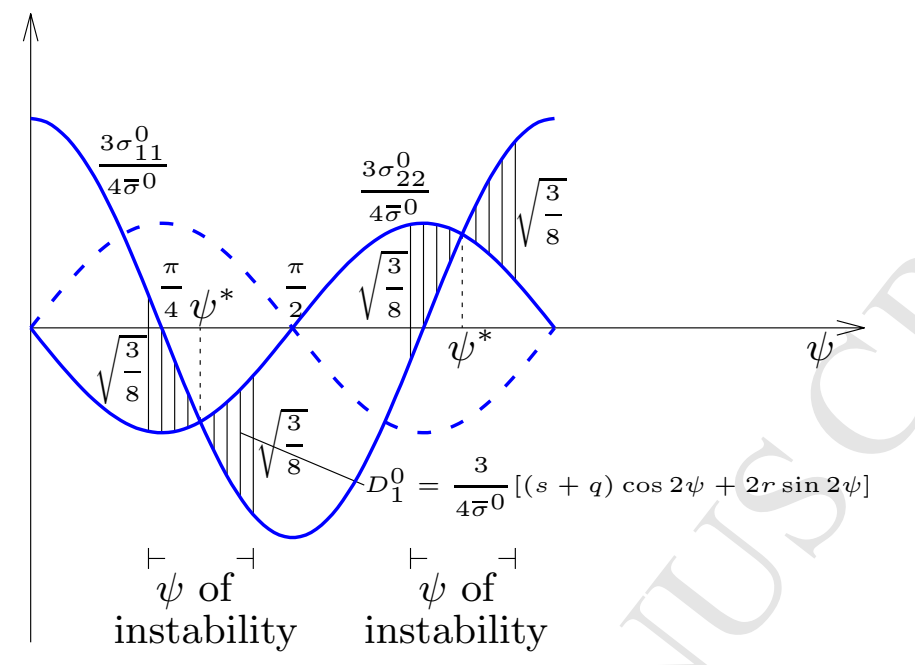

Figure 4: Orientation angles susceptible to given instabilities

loaded in shear. This was observed in thin alternating layers of $\mathrm{Cu}$ and $\mathrm{Nb}$ on Si(100) substrates, as well as in metal-ceramic nanolayered composites (Shen et al., 2012). For the critical angle, the instability condition becomes simply

$$
\frac{\frac{c *|\alpha|}{\theta^{0}}}{\frac{n}{p^{0} \bar{\sigma}^{0}}}>1,
$$

and the maximum instability parameter is given by

$$
X=\frac{\frac{c *|\alpha|}{\theta^{0}}-\frac{n}{p^{0} \bar{\sigma}^{0}}}{\frac{m}{\bar{\sigma}^{0}}} .
$$

However, it is not clear the meaning of (3.48). In the next section, we will prove that this condition is just a criterion for the onset of stress relaxation at which the slope of $s(., t)$ becomes zero.

Finally, we easily conclude that: (i) if $s, r, q>0$, then $\psi^{*}>\frac{\pi}{4}$, (ii) if $s+q \rightarrow 0$ or $r \rightarrow \infty$, then $\psi^{*} \rightarrow 0$ and (iii) if $s+q \rightarrow \infty$ or $r \rightarrow 0$, then 
$\psi^{*} \rightarrow \frac{\pi}{4}$

\section{Non-linear instability analysis}

In this section we will present a non-linear instability analysis that allows to define the onset and evolution of instability as a continuous process. In the sequel, we neglect, for simplicity, the superscript ${ }^{0}$ in the reference solution. Additionally, the stress $r$ is assumed to be steady. By differentiating with respect to the time the integral equation (3.28) we find the integrodifferential equation

$$
\begin{aligned}
\frac{1}{s+q}\left\{1-(1-m) \frac{\frac{4 r^{2}}{(s+q)^{2}}}{\left.1+\frac{4^{2}}{(s+q)^{2}}\right\} \frac{\partial s}{\partial t}}\right. & =\alpha \theta^{-1} \frac{\partial \theta}{\partial t}+n p^{-1} \frac{\partial p}{\partial t} \\
& =\left(\alpha \theta^{-1} c^{*} \sigma+n p^{-1}\right) \dot{p}
\end{aligned}
$$

where $\theta, \frac{\partial \theta}{\partial t}, p, \frac{\partial p}{\partial t}$ are given from temperature (3.26),(3.23), accumulated strain (3.27) and equivalent strain rate expressions (3.24) in terms of $s$. We assume that the initial conditions are such that

$$
n p(y, 0)^{-1}>|\alpha| \theta(y, 0)^{-1} c^{*} \bar{\sigma}
$$

The left-hand side of the evolution equation of lateral stress (4.1) is always positive since $m<1$, therefore the slope of the lateral stress becomes negative if the right-hand side becomes negative, from which, using energy (3.23), equivalent strain rate (3.24) and equivalent stress (3.12) equations and recalling the lower bound of strain (3.21), we obtain again criterion (3.48) of the linearized analysis, but now we know that this criterion corresponds to negative slopes of the lateral stress. Moreover, we can now examine when the zero slope of $s$ corresponds to a maximum value. 
Lemma: Condition $\frac{\frac{c *|\alpha|}{\theta_{0}}}{\frac{n}{p^{0} \bar{\sigma}}}=1$ implies necessarily a condition of maximum $s$ provided that $n<|\alpha|$

In order to examine the conditions for maximum $s$, we take the partial derivative with respect to time in the evolution equation (4.1) and obtain

$$
A(s) \frac{\partial^{2} s}{\partial t^{2}}=B(s)\left[\frac{\partial s}{\partial t}\right]^{2}-C(s) \frac{\partial s}{\partial t}+|\alpha| \theta^{-2}\left[\frac{\partial \theta}{\partial t}\right]^{2}-n p^{-2}(s)\left[\frac{\partial p(s)}{\partial t}\right]^{2},
$$

or

$$
A(s) \frac{\partial^{2} s}{\partial t^{2}}=B(s)\left[\frac{\partial s}{\partial t}\right]^{2}-C(s) \frac{\partial s}{\partial t}+|\alpha| \theta^{-2}(s)\left[c^{*} \bar{\sigma}(s) \dot{p}(s)\right]^{2}-n p^{-2}(s) \dot{p}(s)^{2},
$$

where $A(s), B(s), C(s)$ are functions given in Appendix $\mathrm{C}$ and $\theta, \frac{\partial \theta}{\partial t}, p, \frac{\partial p}{\partial t}$ are obtained from temperature (3.26), energy (3.23), accumulated strain (3.27) and equivalent strain rate (3.24) in terms of $s$.

At the critical time $t=\tilde{t}$, where $\frac{\partial s}{\partial t}=0$ and condition $\frac{\frac{c *|\alpha|}{\frac{\theta}{p}}}{\frac{n}{p \tilde{\sigma}}}=1$ holds, equation (4.4) gives

$$
A(s(\tilde{t})) \frac{\partial^{2} s}{\partial t^{2}}(\tilde{t})=|\alpha| \theta^{-2}(s(\tilde{t}))\left[c^{*} \bar{\sigma}(s(\tilde{t})) \dot{p}(s(\tilde{t}))\right]^{2}-n p^{-2}(s(\tilde{t})) \dot{p}(s(\tilde{t}))^{2},
$$

from which we conclude that, if $n<|\alpha|$, the right-hand member of (4.5) is negative since

$$
\frac{\left[c^{*} \bar{\sigma}(s(\tilde{t}))\right]^{2} p^{2}(\tilde{t})}{\theta^{2}(\tilde{t})}=\left(\frac{n^{2}}{\alpha^{2}}\right)<\frac{n}{|\alpha|} .
$$

Hence, $s(\tilde{t})$ is the maximum value of the lateral stress for all materials with thermal softening larger than strain hardening. In other words, the mechanism of instability is related to the relaxation of $s$. It is natural to conclude that, using the numerical solution for $s$, we can define the onset of instability regime by computing the time $\tilde{t}$ at which $s$ becomes maximum. 
Note 2: Using equivalent strain rate (3.24) and

$$
\ddot{p}=-\frac{8}{\sqrt{3}} r^{2} \frac{\dot{p}}{(s+q)^{3}} \frac{\partial s}{\partial t}
$$

and recalling the evolution equation of accumulated strain (4.12) leads to the time evolution of $s, p, \dot{p}$ at a point, shown in Figure 5, corresponding to the numerical example presented in Section 7.

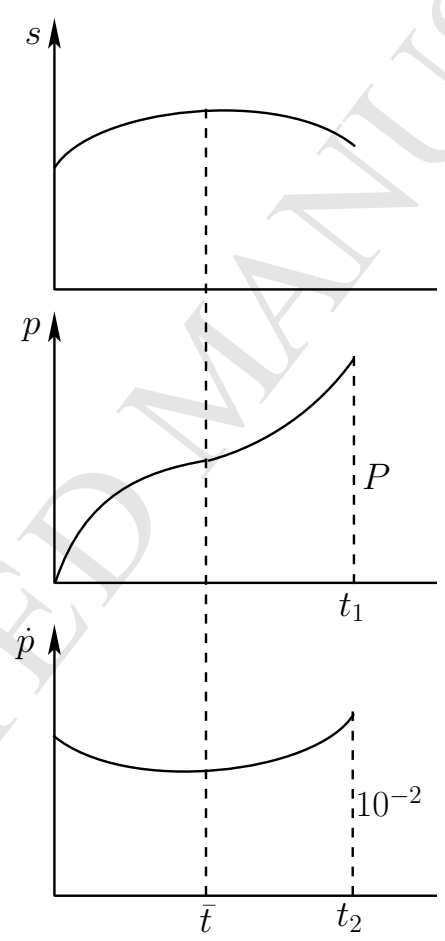

Figure 5: Schematic of evolution of lateral stress, equivalent strain and equivalent strainrate. Critical time: $\min \left\{t_{1}, t_{2}\right\}$

Note 3: From the evolution equation of lateral stress (4.1) we deduce that, for positive initial slope of the lateral stress and for a prescribed value of maximum desirable strain $P$, a sufficient shear stress that excludes instability 
satisfies the inequality

$$
|r|<\frac{4 n\left[\min _{y} \theta_{0}(y)\right]}{|\alpha| c^{*} P},
$$

additionally to the quasi-static condition.

Before passing to the numerical solution, we give an alternative formulation by a non-linear initial-value problem of ordinary differential equation satisfied by the equivalent strain $p(., t)$, that will be used in the sequel for the appropriate initial conditions. To this end, using shear strain (3.17), (3.18) and effective stress (3.19) and recalling energy equation (2.11) and constitutive law (2.12) we express temperature rate and temperature in terms of plastic strain and strain-rate only,

$$
\begin{aligned}
\frac{\partial \theta}{\partial t} & =\sqrt{3} c^{*}|r| \frac{\dot{p}}{\sqrt{1-\frac{4}{3}\left(\frac{v}{\dot{p}}\right)^{2}}} \operatorname{sgn}(r) \\
\theta^{\alpha} & =\frac{1}{G} \sqrt{3}|r| \frac{p^{-n} \dot{p}^{-m}}{\sqrt{1-\frac{4}{3}\left(\frac{v}{\dot{p}}\right)^{2}}}
\end{aligned}
$$

From the expression of the temperature (4.10) by differentiation

$$
\frac{\partial \theta}{\partial t}=\theta\left[-\frac{n}{\alpha} \frac{\dot{p}}{p}-\frac{m}{\alpha} \frac{\ddot{p}}{\dot{p}}-\frac{1}{\alpha} \frac{\frac{4}{3}\left(\frac{v}{\dot{p}}\right)^{2}}{\left(1-\frac{4}{3}{\frac{v^{2}}{\dot{p}}}^{2}\right)} \frac{\ddot{p}}{\dot{p}}\right]
$$

Combining temperature rate (4.9) and (4.11) we arrive at the evolution equation for the equivalent plastic strain

$$
C_{1}(\dot{p}) \frac{\ddot{p}}{\dot{p}}+C_{2}(p, \dot{p}) \frac{\dot{p}}{p}=0
$$

where

$$
C_{1}(\dot{p})=1+K_{2} \frac{\frac{4}{3}\left(\frac{v}{\dot{p}}\right)^{2}}{1-\frac{4}{3}\left(\frac{v}{\dot{p}}\right)^{2}}
$$




$$
\begin{gathered}
C_{2}(p, \dot{p})=K_{1}-K_{3} \frac{p^{1-\frac{n}{|\alpha|}}}{\dot{p}^{\frac{m}{|\alpha|}\left[1-\frac{4}{3}\left(\frac{v}{\dot{p}}\right)^{2}\right]^{\frac{1+|\alpha|}{|\alpha|}}},} \\
K_{1}=\frac{n}{m}, \quad K_{2}=\frac{1}{m}, \quad K_{3}=\frac{|\alpha|}{m} c^{*}(\sqrt{3} r)^{\frac{1+|\alpha|}{|\alpha|}}\left(\frac{1}{G}\right)^{\frac{1}{|\alpha|}}
\end{gathered}
$$

The evolution equation can eventually be solved numerically for any $y$ under initial conditions for $p, \dot{p}$,

$$
p(y, 0)=p_{0}(y), \quad \dot{p}(y, 0)=\dot{p}_{0}(y) .
$$

We note that the linear version of (4.12) with constant $C_{1}, C_{2}$ admits the solution

$$
\tilde{p}(y, t)=\left[(1+\lambda) \dot{p}_{0}(y) p_{0}^{\lambda}(y) t+p_{0}^{1+\lambda}(y), \quad \lambda=\frac{C_{2}}{C_{1}} .\right.
$$

\section{Numerical solution}

The constitutive law (3.22), the energy balance (3.23) and the evolution of the accumulated plastic strain (3.24) can be re-expressed as

$$
\begin{gathered}
s+q=\frac{4 G v}{3} \theta^{\alpha} p^{n} \dot{p}^{m-1}, \\
(s+q)-\frac{1}{c^{*} v} \dot{\theta}+\frac{4 r^{2}}{s+q}=0, \\
\left(1-\frac{3}{4 v^{2}} \dot{p}^{2}\right)+\frac{4 r^{2}}{(s+q)^{2}}=0 .
\end{gathered}
$$

Substituting (5.1) in (5.2) and (5.3) yields

$$
\begin{aligned}
\Phi_{1}(p, \dot{p}, \theta, \dot{\theta}) & =\frac{4 G v}{3} \theta^{\alpha} p^{n} \dot{p}^{m-1}+\frac{3 r^{2}}{G v} \theta^{-\alpha} p^{-n} \dot{p}^{1-m}-\frac{1}{c^{*} v} \dot{\theta}=0, \\
\Phi_{2}(p, \dot{p}, \theta) & =\frac{9 r^{2}}{4 G^{2} v^{2}} \theta^{-2 \alpha} p^{-2 n} \dot{p}^{2-2 m}-\frac{3}{4 v^{2}} \dot{p}^{2}+1=0 .
\end{aligned}
$$


The above system can be solved with the Newton-Raphson scheme, through proper linearization. Setting

$$
p^{\text {updated }}=p+\delta p, \quad \theta^{\text {updated }}=\theta+\delta \theta
$$

leads to the linearized system

$$
\begin{array}{r}
\Phi_{1}+\left(\frac{\partial \Phi_{1}}{\partial p}+\frac{1}{\Delta t} \frac{\partial \Phi_{1}}{\partial \dot{p}}\right) \delta p+\left(\frac{\partial \Phi_{1}}{\partial \theta}-\frac{1}{c^{*} v \Delta t}\right) \delta \theta=0 \\
\Phi_{2}+\left(\frac{\partial \Phi_{2}}{\partial p}+\frac{1}{\Delta t} \frac{\partial \Phi_{2}}{\partial \dot{p}}\right) \delta p+\frac{\partial \Phi_{2}}{\partial \theta} \delta \theta=0
\end{array}
$$

where

$$
\begin{aligned}
\frac{\partial \Phi_{1}}{\partial p} & =\frac{4 G v n}{3} \theta^{\alpha} p^{n-1} \dot{p}^{m-1}-\frac{3 r^{2} n}{G v} \theta^{-\alpha} p^{-n-1} \dot{p}^{1-m} \\
\frac{\partial \Phi_{1}}{\partial \dot{p}} & =-\frac{4 G v(1-m)}{3} \theta^{\alpha} p^{n} \dot{p}^{m-2}+\frac{3 r^{2}(1-m)}{G v} \theta^{-\alpha} p^{-n} \dot{p}^{-m} \\
\frac{\partial \Phi_{1}}{\partial \theta} & =\frac{4 G v \alpha}{3} \theta^{\alpha-1} p^{n} \dot{p}^{m-1}-\frac{3 r^{2} \alpha}{G v} \theta^{-\alpha-1} p^{-n} \dot{p}^{1-m} \\
\frac{\partial \Phi_{2}}{\partial p} & =-\frac{9 r^{2} n}{2 G^{2} v^{2}} \theta^{-2 \alpha} p^{-2 n-1} \dot{p}^{2-2 m} \\
\frac{\partial \Phi_{2}}{\partial \dot{p}} & =\frac{9 r^{2}(2-2 m)}{4 G^{2} v^{2}} \theta^{-2 \alpha} p^{-2 n} \dot{p}^{1-2 m}-\frac{3}{2 v^{2}} \dot{p} \\
\frac{\partial \Phi_{2}}{\partial \theta} & =-\frac{9 r^{2} \alpha}{2 G^{2} v^{2}} \theta^{-2 \alpha-1} p^{-2 n} \dot{p}^{2-2 m} .
\end{aligned}
$$

Setting

$$
\begin{aligned}
A & =\frac{\partial \Phi_{1}}{\partial p}+\frac{1}{\Delta t} \frac{\partial \Phi_{1}}{\partial \dot{p}} \\
B & =\frac{\partial \Phi_{1}}{\partial \theta}-\frac{1}{c^{*} v \Delta t} \\
\Gamma & =\frac{\partial \Phi_{2}}{\partial p}+\frac{1}{\Delta t} \frac{\partial \Phi_{2}}{\partial \dot{p}} \\
\Delta & =\frac{\partial \Phi_{2}}{\partial \theta}
\end{aligned}
$$


The solution of the linearized system reads

$$
\begin{aligned}
\delta p & =\frac{-\Delta \Phi_{1}+B \Phi_{2}}{A \Delta-B \Gamma}, \\
\delta \theta & =\frac{\Gamma \Phi_{1}-A \Phi_{2}}{A \Delta-B \Gamma} .
\end{aligned}
$$

At $t=0$ and with known $p(y, 0)$ and $\theta(y, 0)$, equation $(5.4)_{b}$ is solved with Newton-Raphson for the unknown $\dot{p}(y, 0)$ :

$$
\Phi_{2}^{0}+\frac{\partial \Phi_{2}^{0}}{\partial \dot{p}} \delta \dot{p}=0 \quad \text { or } \quad \delta \dot{p}=-\frac{\Phi_{2}^{0}}{\partial \Phi_{2}^{0} / \partial \dot{p}} .
$$

To start the Newton-Raphson scheme, a good initial prediction for the $\dot{p}$ can be obtained by considering $2-2 m \approx 2$. With this assumption, equation $(5.4)_{b}$ gives

$$
\dot{p}^{\text {pred }}(y, 0)=\frac{1}{\sqrt{\frac{3}{4 v^{2}}-\frac{9 r^{2}}{4 G^{2} v^{2}} \theta^{-2 \alpha} p^{-2 n}}} .
$$

In order to apply the numerical scheme we need to recall the following conditions imposed to initial data.

Compatibility condition for initial $s(y, t): s(y, 0)=s_{0}(y)$ is given from (3.34).

Compatibility condition for initial equivalent strain rate:

$$
\dot{p}_{0}(y)=\frac{2}{\sqrt{3}} v \sqrt{1+4 \frac{r^{2}}{\left(s_{0}(y)+q\right)^{2}}} .
$$

Possible condition for positive slop of $\mathrm{s}(\mathrm{y}, 0)$ : From (4.1)

$$
s_{0}(y)+q<\sqrt{A}=\sqrt{\frac{4 n^{2} \theta_{0}(y)^{2}}{\alpha^{2}\left(c^{*}\right)^{2} p_{0}(y)^{2}}-4 r^{2}} .
$$


Necessary condition for positive A (maximum shearing):

$$
|r|<\frac{4 n\left[\min _{y} \theta_{0}(y)\right]}{\alpha c^{*}\left[\max _{y} p_{0}(y)\right]}
$$

Possible condition for $\ddot{p}(y, 0)<0$ : From (4.12)

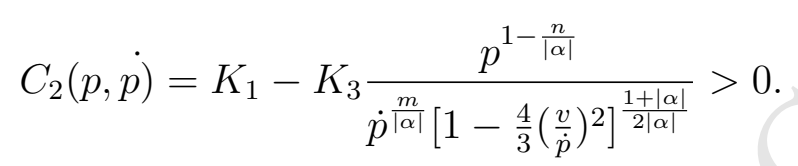

Necessary condition for minimum strain rate: From $s+q=\frac{r}{\sqrt{\frac{3 \dot{p}^{2}}{16 v^{2}}-\frac{1}{4}}}>0$

$$
\dot{p}_{0}(y)>1.155 \mathrm{v} .
$$

Necessary condition for maximum strain rate $\dot{p}(y, t) \leq 10^{-2}$

$$
\frac{s_{0}(y)+q}{r}>0.233
$$

This numerical scheme is utilized for obtaining the basic solution that is going to be utilized for both the instability analysis and the homogenization results. For the homogenization, the obtained results are going to be compared with those from the asymptotic expansion homogenization theory for thermomechanical processes, as described in Chatzigeorgiou et al. (2018). The theoretical and numerical details of the the asymptotic expansion homogenization theory are briefly presented in Appendix D.

\section{Homogenized problem}

In this section, we consider the case of a periodically graded material, with a very fine period, such that all given and unknown functions $\psi$ can be expressed as functions of both the macro- $y$ and micro-coordinates $\bar{y}=\frac{y}{\epsilon}$ as

$$
\psi^{\epsilon}(y) \doteq \psi(y, \bar{y})
$$


being periodic with respect to $\bar{y}$ with period $\bar{Y}$. Then, by considering $\epsilon \rightarrow 0$, all techniques adopted by the periodic homogenization can be applied, including weak limits of bounded functions, in order to define the homogenized functions of the heterogeneous problem

$$
\begin{gathered}
v_{x}^{\epsilon}=v x+\phi^{\epsilon}(y, t), \quad v_{y}=-v y, \\
\bar{\sigma}^{\epsilon}(y, t)=\sqrt{\frac{3}{4}\left(s^{\epsilon}(y, t)+q\right)^{2}+3 r^{2},} \\
\dot{p}^{\epsilon}=\frac{4}{3} \frac{v \bar{\sigma}^{\epsilon}(y, t)}{s^{\epsilon}(y, t)+q}, \\
\dot{\varepsilon}_{x y}^{\epsilon}=\frac{1}{2} \frac{\partial \phi(y, t)}{\partial t}=\frac{2 v r}{s^{\epsilon}(y, t)+q}, \\
\frac{\partial \theta^{\epsilon}}{\partial t}=c^{* \epsilon} \bar{\sigma}^{\epsilon} \dot{p}^{\epsilon}, \\
\bar{\sigma}^{\epsilon}=G^{\epsilon} \theta^{\alpha \epsilon} p^{n \epsilon} \dot{p}^{m \epsilon} .
\end{gathered}
$$

The heterogeneous material may be a functionally periodic material or a multilayered material with continuously graded interfaces. For a multilayer, all given properties are obtained by alternate piecewise constant and smoothly connected periodic functions (see Figure 6 for the evolution of the parameter $G)$.

In the sequel, we denote by $\left\langle\psi^{\epsilon}\right\rangle$ the average of a periodic function $\psi^{\epsilon}(y)$ over its period

$$
<\psi^{\epsilon}(y)>\doteq \frac{1}{\bar{Y}} \int_{0}^{\bar{Y}} \psi(y, \bar{y}) d \bar{y},
$$

so the weak limit of every bounded periodic function $\psi^{\epsilon}(y),-\frac{h}{2} \leq y \leq \frac{h}{2}$ 


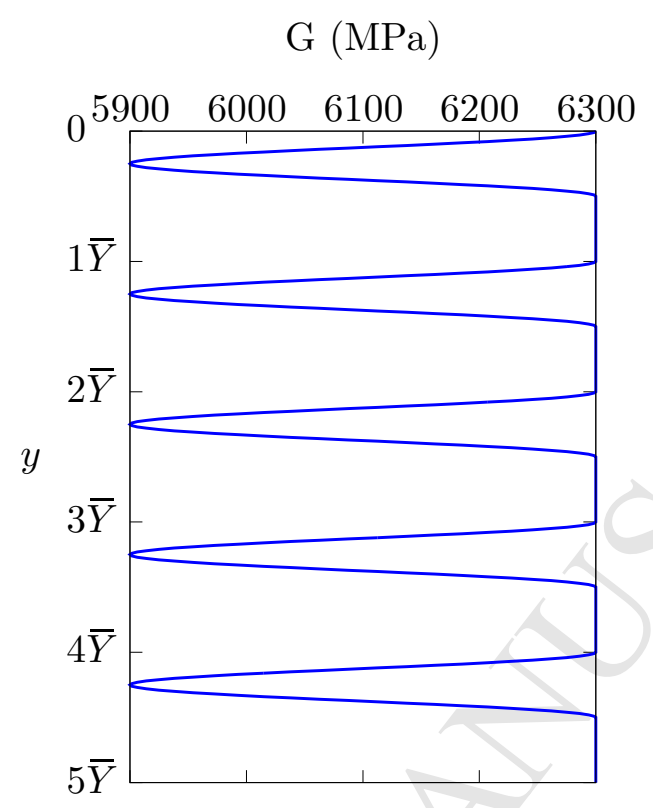

Figure 6: Example of material property

for $\epsilon \rightarrow 0$ is given by

$$
\int_{-\frac{h}{2}}^{\frac{h}{2}} \psi^{\epsilon}(y) f(y) d y \rightarrow \int_{-\frac{h}{2}}^{\frac{h}{2}} \frac{1}{\bar{Y}} \int_{0}^{\bar{Y}} f(y) \psi(y, \bar{y}) d \bar{y} d y, \quad \forall f(y) \in C\left[-\frac{h}{2}, \frac{h}{2}\right] .
$$

This is a non-linear homogenization problem, in which non-linear equations, as well as products of bounded sequences do not converge weakly, so the homogenized solution must rely on the numerical solution $p^{\epsilon}(., t)$ and $\theta^{\epsilon}(, t)$ at every time $t$ and for fixed small $\epsilon$ and the corresponding values of $s^{\epsilon}(., t)$. The homogenized structure is loaded by $q, r$ and $w=<s^{\epsilon}(y, t)>$ (see Figure 7).

In Figure 8 we see a picture of the velocity field of four points forming a rectangle in the undeformed situation, as well as the unknown velocity 


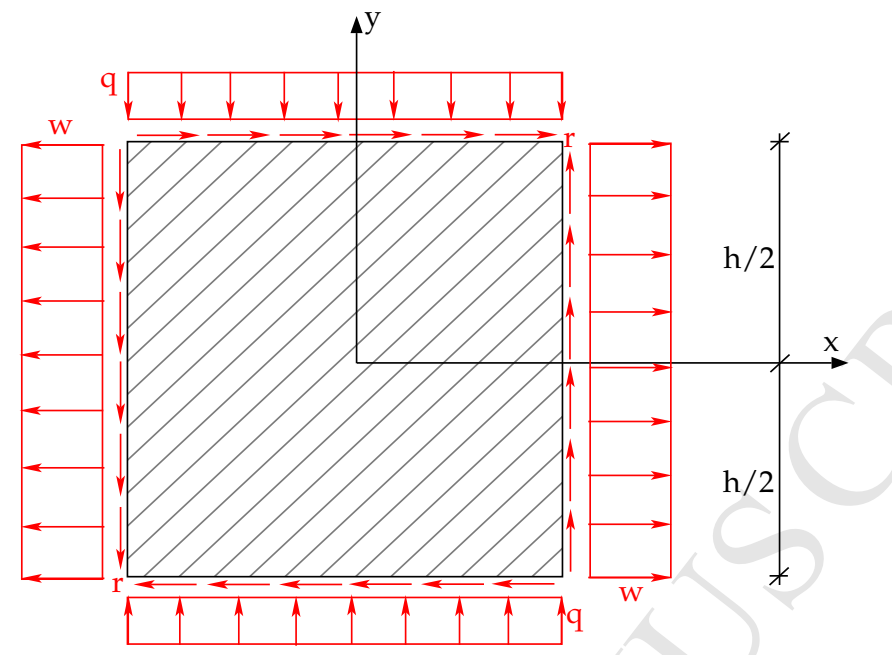

Figure 7: Homogenized lateral stress and structure

function $\phi(y, t)$ in (3.9) at a time $t$, having a given value at $y=0$.

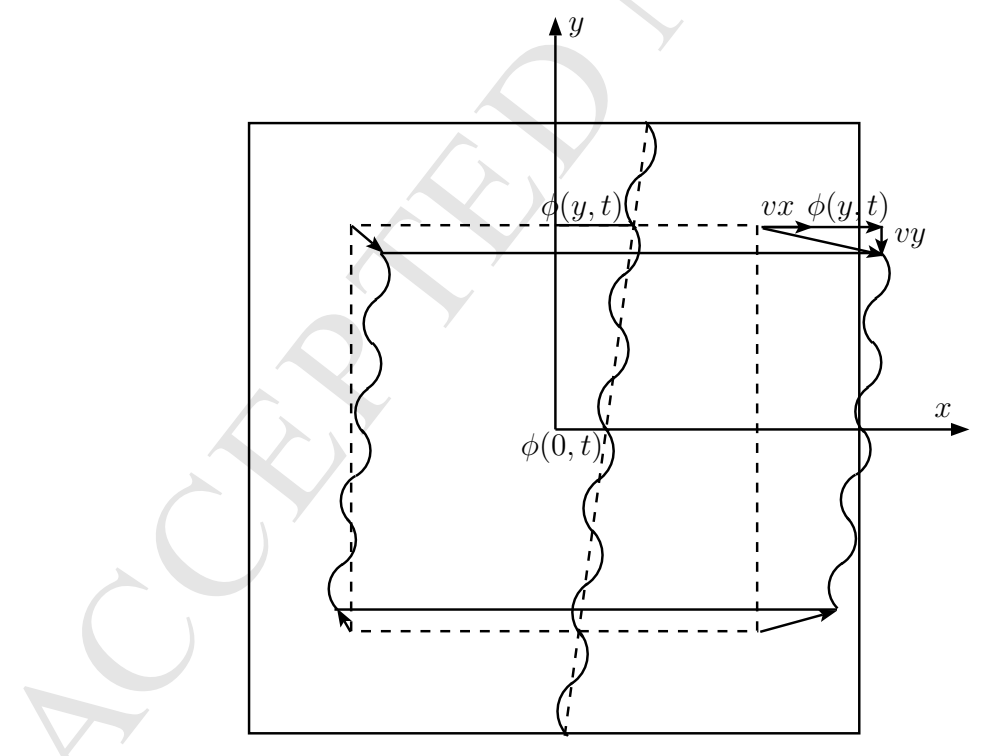

Figure 8: Unknown velocity function is not periodic, but its derivative is periodic

Note that $\phi(y,$.$) is not periodic, but its derivative \frac{\partial \phi}{\partial t}(y,$.$) is periodic, so$ 
$\phi(y,$.$) can be decomposed into a linear and a periodic part \omega(y,),. \omega\left( \pm \frac{h}{2}, t\right)=$ 0 as

$$
\phi^{\epsilon}(y, t)=\phi(0, t)+\int_{0}^{y} \frac{\partial \phi^{\epsilon}(\xi, t)}{\partial y} d \xi=\alpha y+\phi(0, t)+\omega^{\epsilon}(y, t),
$$

therefore

$$
<\frac{\partial \phi^{\epsilon}(\xi, t)}{\partial y}>=\alpha+<\frac{\partial \omega^{\epsilon}(y, t)}{\partial t}>=\alpha .
$$

On the other hand, from shear strain (6.5), the macro-shear strain-rate is given by

$$
\dot{E}_{x y}=<\dot{\varepsilon}_{x y}^{\epsilon}>=\frac{1}{2}<\frac{\partial \phi(y, t)}{\partial y}>=2 v r<\frac{1}{s^{\epsilon}(y, t)+q}>.
$$

Combining equivalent strain rate (6.4) and energy (6.6) equations, we deduce the dissipation in terms of the lateral stress

$$
D^{\epsilon}=\frac{1}{c^{* \epsilon}} \frac{\partial \theta^{\epsilon}}{\partial t}=\bar{\sigma}^{\epsilon} \dot{p}^{\epsilon}=v\left(s^{\epsilon}(y, t)+q\right)+4 v r^{2} \frac{1}{s^{\epsilon}(y, t)+q} .
$$

from which, by averaging, the homogenized dissipation

$$
\begin{aligned}
D & =\left\langle D^{\epsilon}>=<\frac{1}{c^{* \epsilon}} \frac{\partial \theta^{\epsilon}}{\partial t}>\right. \\
& =\left\langle\bar{\sigma}^{\epsilon} \dot{p}^{\epsilon}>=v<s^{\epsilon}(y, t)+q>+4 v r^{2}<\frac{1}{s^{\epsilon}(y, t)+q}>.\right.
\end{aligned}
$$

This can be written in terms of macro-shear strain-rate and macro-lateral stress as

$$
D=<D^{\epsilon}>=<\bar{\sigma}^{\epsilon} \dot{p}^{\epsilon}>=v(w+q)+r \dot{E}_{x y} .
$$

This is a rare example of homogenized dissipation that can be expressed in relatively explicit form, thanks to the special solution of the problem and the form of boundary conditions. 


\section{Numerical example}

In this section we present a numerical example of a multilayer with graded interfaces.

We note that the initial data and the boundary conditions are chosen such that necessary conditions (3.34), (5.12), (5.14), (5.15) are respected. The case of compressive initial lateral stress is not treated, following the same lines. We note that, in this case, an additional investigation for buckling of interfaces using data of heterogeneous lateral stress could eventually be of interest.

Data:

$$
\begin{aligned}
& r=180 \mathrm{MPa}, \\
& q=200 \mathrm{MPa}, \\
& v=0.0011 / \mathrm{s}, \\
& p(y, 0)=0.02, \\
& \theta(y, 0)=300 \mathrm{~K},
\end{aligned}
$$

$$
\begin{gathered}
G(y)=\left\{\begin{array}{cl}
6100+200 \cos \left(\frac{2 \pi}{f \bar{Y}} y\right), & 0 \leq y \leq f \bar{Y}, \\
6300, & f \bar{Y} \leq y \leq \bar{Y},
\end{array} \quad \mathrm{MPa},\right. \\
m(y)=\left\{\begin{array}{cc}
0.017+0.002 \cos \left(\frac{2 \pi}{f \bar{Y}} y\right), & 0 \leq y \leq f \bar{Y} \\
0.019, & f \bar{Y} \leq y \leq \bar{Y},
\end{array}\right. \\
\alpha(y)=\left\{\begin{array}{cc}
-0.34-0.04 \cos \left(\frac{2 \pi}{f \bar{Y}} y\right), & 0 \leq y \leq f \bar{Y}, \\
-0.38, & f \bar{Y} \leq y \leq \bar{Y},
\end{array}\right.
\end{gathered}
$$




$$
\begin{gathered}
n(y)=\left\{\begin{array}{cc}
0.014+0.001 \cos \left(\frac{2 \pi}{f \bar{Y}} y\right), & 0 \leq y \leq f \bar{Y}, \\
0.015, & f \bar{Y} \leq y \leq \bar{Y},
\end{array}\right. \\
c^{*}(y)=\left\{\begin{array}{cc}
0.20077+0.03 \cos \left(\frac{2 \pi}{f Y} y\right), & 0 \leq y \leq f \bar{Y}, \\
0.23077, & f \bar{Y} \leq y \leq \bar{Y},
\end{array} \mathrm{~m}^{3} \mathrm{~K} / \mathrm{MJ} .\right.
\end{gathered}
$$

The material parameters are motivated by experimental data available in the literature (see also Chatzigeorgiou et al., 2007). The fraction $f$ is set to 0.5.

We compute $s_{k}$ at two characteristic points, at $y=0$ with the absolute maximum material parameters and at $y=0.25 \bar{Y}$ the absolute minimum material parameters (see Figure 9). The life of the process can be defined as

$$
t=\min \left[t_{1}, t_{2}\right]
$$

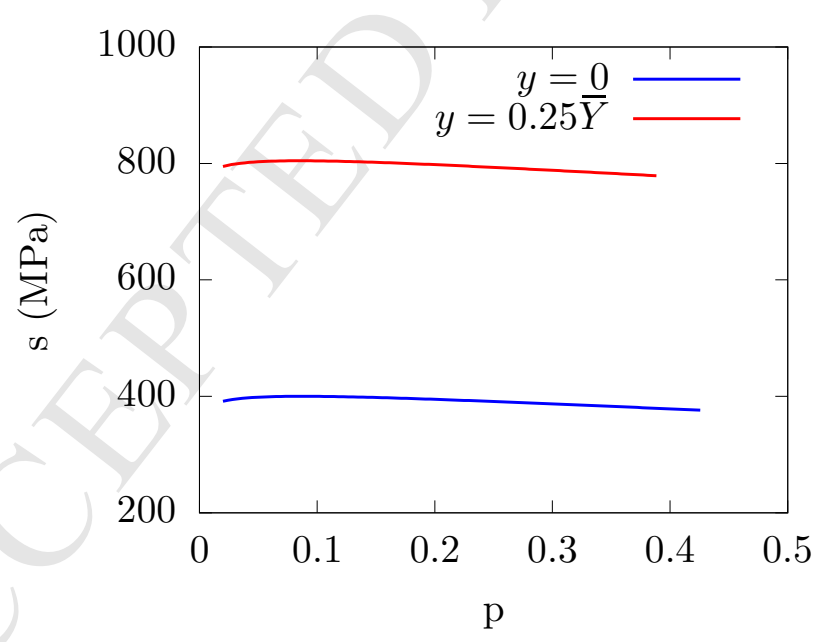

Figure 9: Lateral stress versus equivalent strain. Softening effect

In Figures 10 and 11 we see the evolution in time of $s, p, \dot{p}$ at the two positions, corresponding to the stiffest and weakest regions respectively. In 
Figure 12 we see the oscillating stress and shear strain of the composite at a characteristic time $(t=50 \mathrm{~s})$, exhibiting smooth evolution with respect to the position $y$.

For the homogenization process, we compare the results obtained by the present methodology with those corresponding value computed by the fully coupled thermomechanical asymptotic expansion homogenization theory presented in Chatzigeorgiou et al. (2018) (see details in Appendix D). In Figure $13_{a}$ the time-evolution of the homogenized lateral stress $\mathrm{w}$ presents a maximum value, and the observed maximum difference between the two approaches is of the order of $2 \%$. In Figure $13_{b}$ we see the time-evolution of the homogenized shear strain and we obtain a regime of increasing positive rate after $50 \mathrm{sec}$. A maximum difference of the order of $4 \%$ between the two approaches is observed. Finally, in Figure $13_{c}$, the time-evolution of the homogenized dissipation rate $D(t)$ presents a maximum value at around $t=50$ $\mathrm{s}$ and decreases as the process continues to evolve in the softening regime.

\section{Conclusion}

In this paper, a special solution of the two-dimensional quasistatic problem of a rigid-thermo-visco-plastic heterogeneous plate under biaxial compression/tension or compression/compression combined with shearing, that preserves prescribed uniform normal strains for manufacturing purposes, is proposed. An instability analysis is presented that reveals that the onset of instability depends on the time-variation of the heterogeneous lateral stress. 


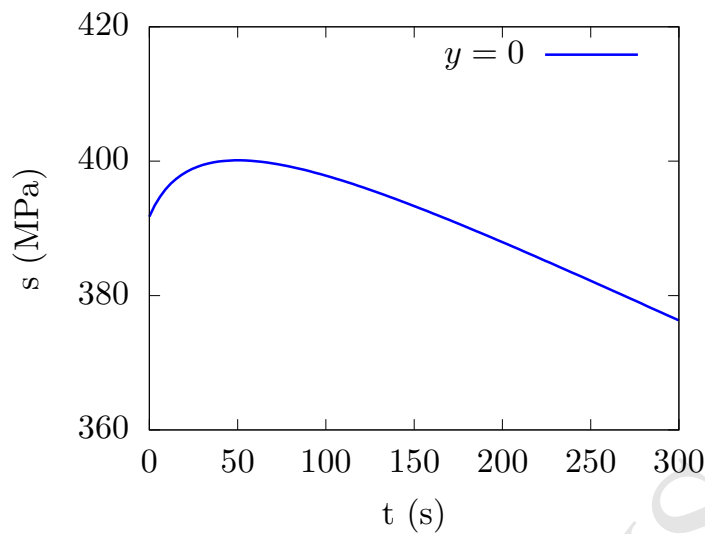

(a)

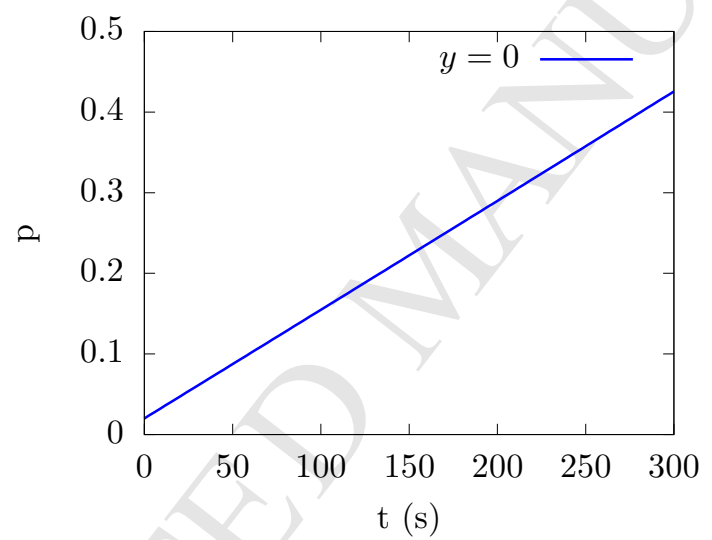

(b)

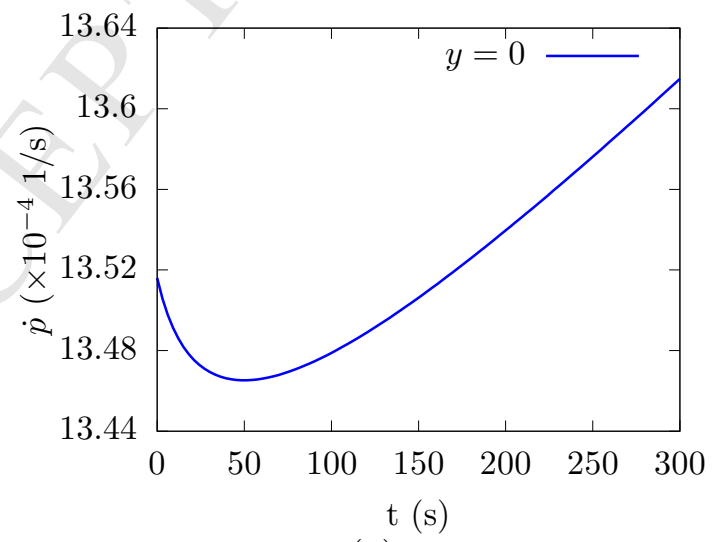

(c)

Figure 10: Evolution in time of $s, p, \dot{p}$ at $y=0$ 


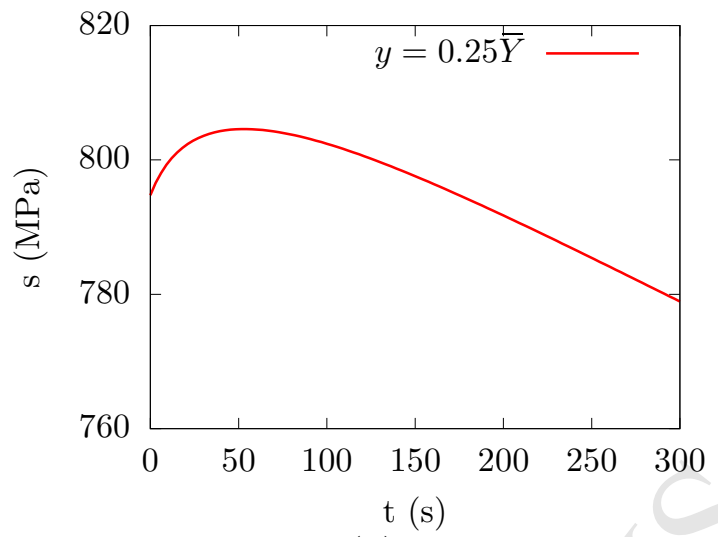

(a)

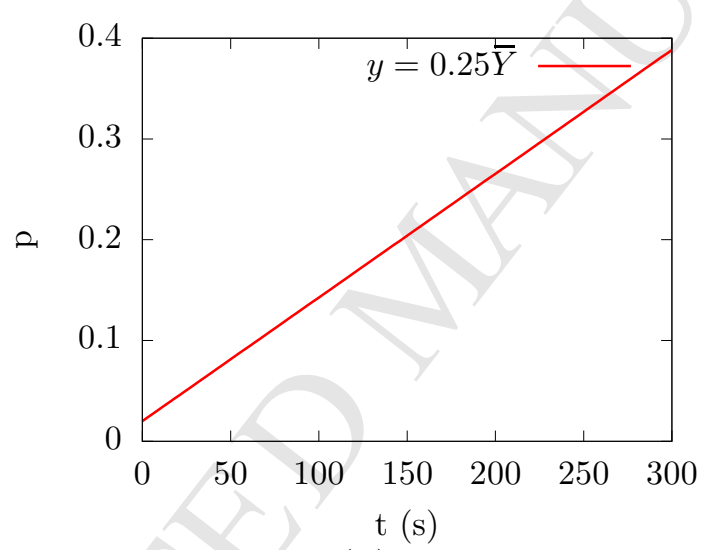

(b)

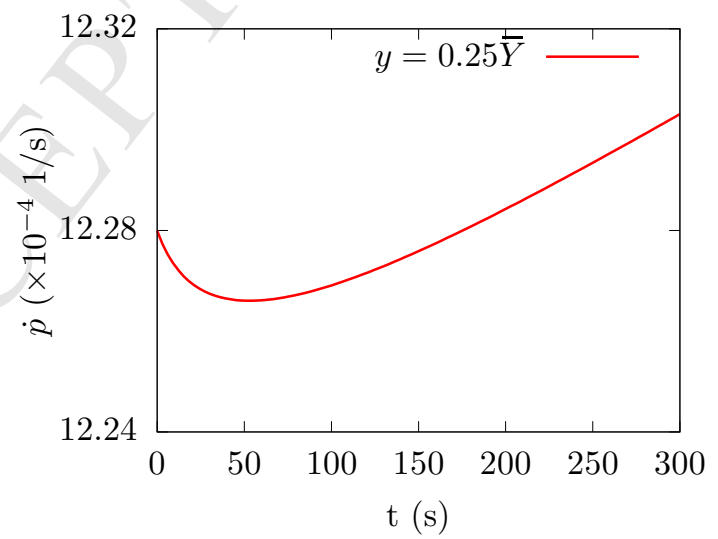

(c)

Figure 11: Evolution in time of $s, p, \dot{p}$ at $y=0.25 \bar{Y}$ 


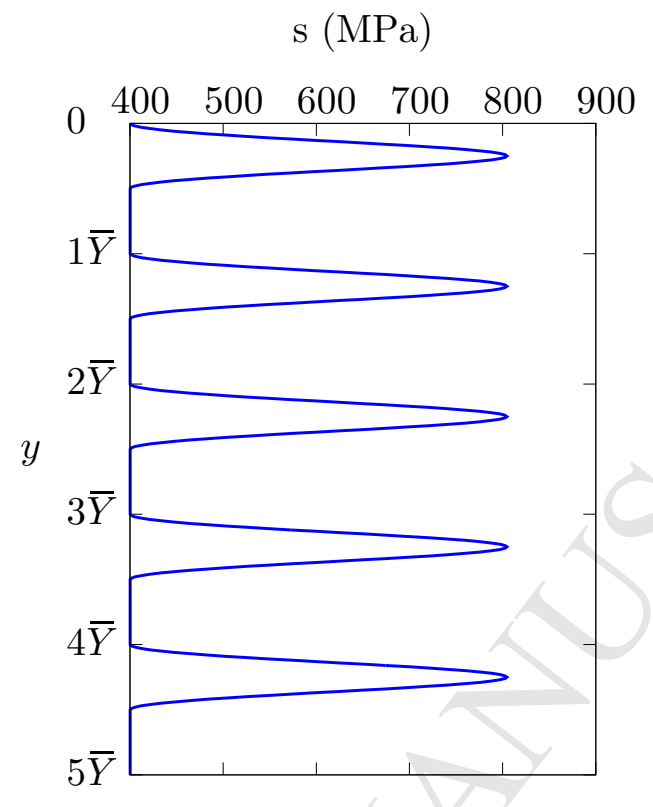

(a)

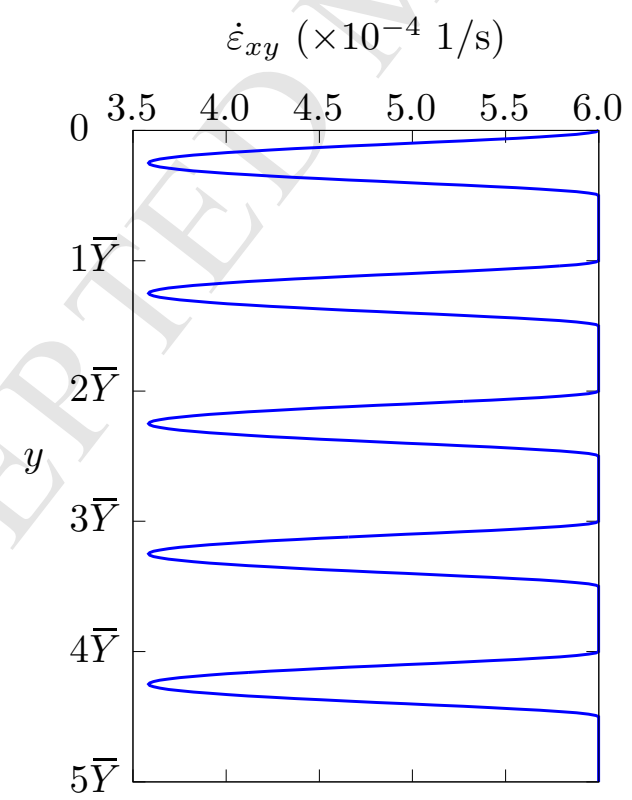

(b)

Figure 12: Distribution of lateral stress and shear strain at $t=50 \mathrm{~s}$. Localization effect 


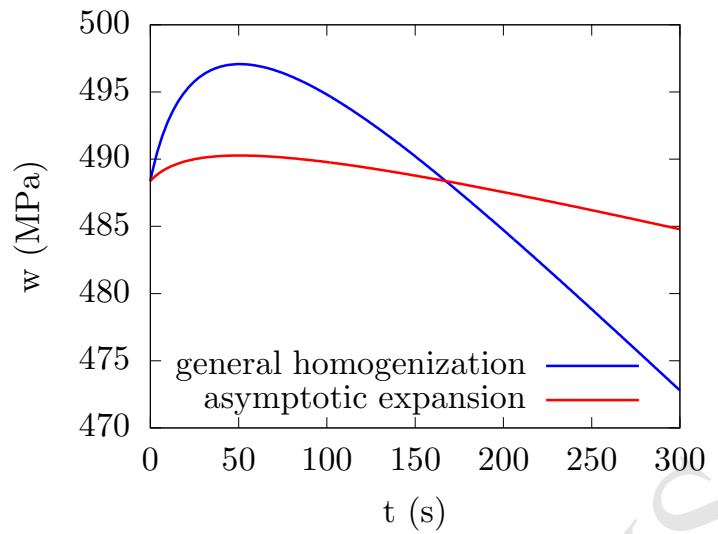

(a)

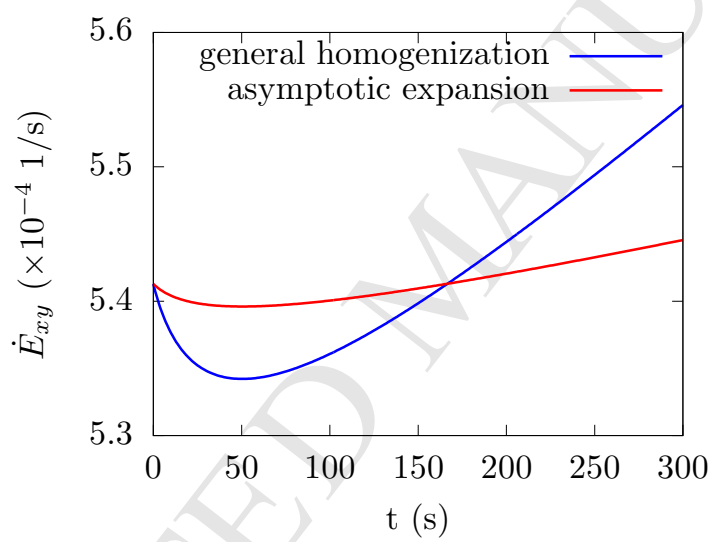

(b)

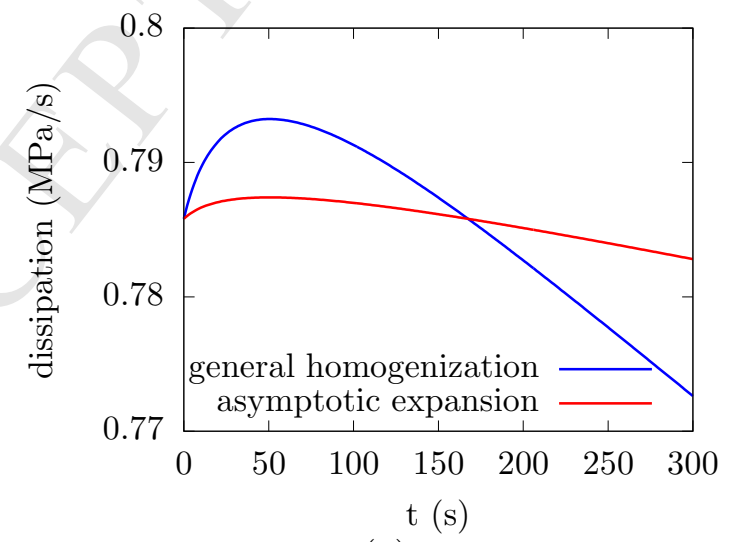

(c)

Figure 13: Evolution of homogenized lateral stress, shear strain and dissipation 
Moreover, the homogenized problem of a periodic multilayer with continuously graded interfaces is presented and the required effective uniform lateral loading, together with the effective shear strain and effective dissipation, are expressed in terms of the lateral stress of the heterogeneous problem. The results on the heterogeneous lateral normal stress could be eventually used for further investigation of high stress concentrations as well as of microbuckling (for compressive lateral stress), while the results on the heterogeneous shear strain distribution could be used for high non-uniformities control.

\section{Appendix A}

We take the logarithm of the constitutive law (2.12), differentiate with respect to time and substitute $\frac{\partial \theta}{\partial t}$ from the energy equation (2.11) to deduce

$$
m \frac{\partial z}{\partial t}+\frac{1}{m \bar{\sigma}} \frac{\partial \bar{\sigma}}{\partial t} z-\left[\alpha\left(\frac{\beta}{\varrho c}\right) \frac{\bar{\sigma}}{\theta}+\frac{n}{p}\right]=0,
$$

where $z=\left(\frac{\partial p}{\partial t}\right)^{-1}$. The solution of (9.1) has the following representation in terms of equivalent stress and temperature level and the thermomechanical parameters:

$$
z(y, t)=e^{-\int P(y, t) d t} \int_{t_{0}}^{t} e^{\int P(y, s) d s} Q(y, \tau) d \tau+e^{-\int P(y, t) d t} z\left(y, t_{0}\right),
$$

where

$$
\begin{aligned}
P(y, t) & =\frac{1}{m(y) \bar{\sigma}(y, t)} \frac{\partial \bar{\sigma}(y, t)}{\partial t}, \\
Q(y, t) & =\frac{\alpha(y)}{m(y)}\left(\frac{\beta}{m(y) \varrho(y) c(y)}\right) \frac{\bar{\sigma}(y, t)}{\theta(y, t)}+\frac{n(y)}{m(y) p(y, t)} .
\end{aligned}
$$

Since $z\left(y, t_{0}>0\right.$ we conclude that, if $Q(\hat{y}, t)<0$ for some material point $\hat{y}$ after a strain and loading level, then for this point there exists a critical 
time at which the equivalent strain-rate increases without bound (see also Chatzigeorgiou and Charalambakis, 2005; Chatzigeorgiou et al., 2007).

\section{Appendix B}

The determinant of the linearized homogeneous algebraic system takes the form

$$
\left(\begin{array}{ccccccccc}
D_{1}^{0} & -D_{1}^{0} & 2 D_{3}^{0} & -1 & 0 & 0 & 0 & 0 & 0 \\
0 & 0 & 0 & -\frac{x}{\bar{\sigma}^{0}} & 0 & 0 & 0 & \frac{R}{\dot{p}^{0}} & \frac{\alpha X}{\theta^{0}} \\
\frac{D_{11}^{0}}{\bar{\sigma}^{0}} & -\frac{D_{11}^{0}}{\bar{\sigma}^{0}} & \frac{2 D_{13}^{0}}{\bar{\sigma}^{0}} & 0 & -\frac{1}{\dot{p}^{0}} & 0 & 0 & \frac{D_{1}^{0}}{\dot{p}^{0}} & 0 \\
-\frac{D_{11}^{0}}{\bar{\sigma}^{0}} & \frac{D_{11}^{0}}{\bar{\sigma}^{0}} & -\frac{2 D_{13}^{0}}{\bar{\sigma}^{0}} & 0 & 0 & -\frac{1}{\dot{p}^{0}} & 0 & -\frac{D_{1}^{0}}{\dot{p}^{0}} & 0 \\
\frac{D_{13}^{0}}{\bar{\sigma}^{0}} & -\frac{D_{13}^{0}}{\bar{\sigma}^{0}} & \frac{D_{33}^{0}}{\bar{\sigma}^{0}} & 0 & 0 & 0 & -\frac{1}{\dot{p}^{0}} & \frac{D_{1}^{0}}{\dot{p}^{0}} & 0 \\
0 & 0 & 0 & 0 & 0 & \xi^{2} & 1 & 1 & 1 \\
\xi & 0 & 0 & 0 & 0 & 0 & 0 & 0 & 0 \\
0 & 0 & \xi & 0 & 0 & 0 & 0 & 0 & 0 \\
0 & 0 & 0 & c^{*} & 0 & 0 & 0 & \frac{c^{*} \bar{\sigma}^{0}}{\dot{p}^{0}} & -X
\end{array}\right)
$$

where

$$
\begin{gathered}
\sigma_{11}^{0}=s(y, t) \cos ^{2} \psi-q(t) \sin ^{2} \psi+r(t) \sin 2 \psi \\
\sigma_{22}^{0}=s(y, t) \sin ^{2} \psi-q(t) \cos ^{2} \psi-r(t) \sin 2 \psi \\
\sigma_{12}^{0}=-\frac{q(t)+s(y, t)}{2} \sin 2 \psi+r(t) \cos 2 \psi \\
D_{1}^{0}=\frac{3}{4 \bar{\sigma}^{0}}\left(\sigma_{11}^{0}-\sigma_{22}^{0}\right)=\frac{3}{4 \bar{\sigma}^{0}}[(s(y, t)+q(t)) \cos 2 \psi+2 r(t) \sin 2 \psi] \\
D_{2}^{0}=\frac{3}{2 \bar{\sigma}^{0}} \sigma_{12}^{0}=\frac{3}{2 \bar{\sigma}^{0}}[-(s(y, t)+q(t)) \sin 2 \psi+2 r(t) \cos 2 \psi] \\
D_{11}^{0}=\frac{3}{4}-\left(D_{1}^{0}\right)^{2}, \quad D_{13}^{0}=-D_{1}^{0} D_{3}^{0}, \quad D_{33}^{0}=\frac{3}{2}-2\left(D_{3}^{0}\right)^{2}
\end{gathered}
$$




$$
\begin{gathered}
X=\frac{\eta}{\dot{p}^{0}} \\
R=m X+\frac{n}{p^{0}} .
\end{gathered}
$$

Using the von Mises expression (3.12),

$$
\left(D_{1}^{0}\right)^{2}+\left(D_{3}^{0}\right)^{2}=\frac{3}{4}
$$

\section{Appendix $\mathrm{C}$}

$$
\begin{gathered}
A(s)=\frac{1}{s+q}\left\{1-(1-m) \frac{\frac{4 r^{2}}{(s+q)^{2}}}{1+\frac{4^{2}}{(s+q)^{2}}}\right\} \\
B(s)=\frac{1}{(s+q)^{2}}\left\{1-(1-m) \frac{12 r^{2}(s+q)^{2}+16 r^{4}}{\left[(s+q)^{2}+4 r^{2}\right]^{2}}\right\} \\
C(s)=\frac{8 n v r^{2}}{\sqrt{3}} \frac{1}{\sqrt{1+\frac{4 r^{2}}{(s+q)^{2}}}(s+q)^{3}} \frac{1}{p(s)}+|\alpha| c^{*} v\left[1-\frac{4 r^{2}}{(s+q)^{2}} \frac{1}{\theta(s)} .\right.
\end{gathered}
$$

\section{Appendix D}

According to the asymptotic expansion homogenization framework, discussed in Chatzigeorgiou et al. (2018), the heterogeneous problem

$$
\begin{aligned}
\dot{\varepsilon}_{x x}^{\epsilon} & =v, \\
\dot{\varepsilon}_{y y}^{\epsilon} & =-v, \\
\dot{\varepsilon}_{x y}^{\epsilon} & =\frac{2 v r}{s^{\epsilon}+q}, \\
s^{\epsilon} & =\frac{4 G^{\epsilon} v}{3}\left(\theta^{\epsilon}\right)^{\alpha^{\epsilon}}\left(p^{\epsilon}\right)^{n^{\epsilon}}\left(\dot{p}^{\epsilon}\right)^{m^{\epsilon}-1}-q, \\
\dot{p}^{\epsilon} & =v \sqrt{\frac{4}{3}\left(1+\frac{4 r^{2}}{\left(s^{\epsilon}+q\right)^{2}}\right)} \\
\dot{\theta}^{\epsilon} & =c^{* \epsilon} v\left(s^{\epsilon}+q+\frac{4 r^{2}}{\left(s^{\epsilon}+q\right)}\right),
\end{aligned}
$$


leads to the microequations in the unit cell (exponent 0 denotes microquantity, bar denotes macro-quantity)

$$
\begin{aligned}
\dot{\varepsilon}_{x x}^{0} & =v, \\
\dot{\varepsilon}_{y y}^{0} & =-v, \\
\dot{\varepsilon}_{x y}^{0} & =\frac{2 v r}{s^{0}+q}, \\
s^{0} & =\frac{4 G v}{3} \bar{\theta}^{\alpha}\left(p^{0}\right)^{n}\left(\dot{p}^{0}\right)^{m-1}-q, \\
\dot{p}^{0} & =v \sqrt{\frac{4}{3}\left(1+\frac{4 r^{2}}{\left(s^{0}+q\right)^{2}}\right)},
\end{aligned}
$$

and the macroequations

$$
\begin{aligned}
\dot{\bar{\varepsilon}}_{x x} & =\left\langle\dot{\varepsilon}_{x x}^{0}\right\rangle=v, \\
\dot{\bar{\varepsilon}}_{y y} & =\left\langle\dot{\varepsilon}_{y y}^{0}\right\rangle=-v, \\
\dot{\bar{\varepsilon}}_{x y} & =\left\langle\dot{\varepsilon}_{x y}^{0}\right\rangle=2 v r\left\langle\frac{1}{s^{0}+q}\right\rangle, \\
\bar{s} & =\left\langle s^{0}\right\rangle, \\
\dot{\bar{\theta}} & =\left\langle c^{*} v\left(s^{0}+q+\frac{4 r^{2}}{\left(s^{0}+q\right)}\right)\right\rangle .
\end{aligned}
$$

The homogenization calculation scheme is written as follows:

1. At $t=0, \bar{\theta}(0)=\theta^{\text {ref }}$ and $p^{0}(y, 0)=p^{\text {ref }}$ everywhere.

2. Solve the nonlinear equation

$$
\Phi^{0}\left(\dot{p}^{0}\right)=\frac{9 r^{2}}{4 G^{2} v^{2}} \bar{\theta}^{-2 \alpha}\left(p^{0}\right)^{-2 n}\left(\dot{p}^{0}\right)^{2-2 m}-\frac{3}{4 v^{2}}\left(\dot{p}^{0}\right)^{2}+1=0,
$$

and compute $\dot{p}^{0}(y, 0)$.

3. Compute $s^{0}(y, 0)$ from $(12.2)_{4}$.

4. Compute $\dot{\bar{\theta}}$ from $(12.3)_{5}$. 
5. Incremental computational procedure at $t=t_{n+1}=t_{n}+\Delta t$ :

(a) At $t=t_{n}$ everything is known.

(b) $\operatorname{Set} \bar{\theta}\left(t_{n+1}\right)=\bar{\theta}\left(t_{n}\right)+\Delta t \dot{\bar{\theta}}\left(t_{n}\right)$.

(c) Assume

$p^{0}\left(y, t_{n+1}\right)=p^{0}\left(y, t_{n}\right)+\Delta t \dot{p}^{0}\left(y, t_{n}\right), \quad \dot{p}^{0}\left(y, t_{n+1}\right)=\dot{p}^{0}\left(y, t_{n}\right)$,

as prediction, solve iteratively the differential equation

$$
\Phi\left(p^{0}, \dot{p}^{0}\right)=\frac{9 r^{2}}{4 G^{2} v^{2}} \bar{\theta}^{-2 \alpha}\left(p^{0}\right)^{-2 n}\left(\dot{p}^{0}\right)^{2-2 m}-\frac{3}{4 v^{2}}\left(\dot{p}^{0}\right)^{2}+1=0,
$$

and compute $p^{0}\left(y, t_{n+1}\right)$ and $\dot{p}^{0}\left(y, t_{n+1}\right)$.

(d) Compute $s^{0}\left(y, t_{n+1}\right)$ from $(12.2)_{4}$.

(e) Compute $\dot{\bar{\theta}}\left(t_{n+1}\right)$ from $(12.3)_{5}$.

Note: The dissipation is computed by equation (6.15), considering the stress $s$ as calculated by the above iterative scheme.

\section{References}

Anand, L., Kim, K., Shawki, T., 1987. Onset of shear localization in viscoplastic solids. Journal of the Mechanics and Physics of Solids 35(4), 407-429.

Bai, Y., 1982. Thermo-plastic instability in simple shear. Journal of the Mechanics and Physics of Solids 30(4), 195-207.

Bai, Y., Keller, T., 2009. Shear failure of pultruded fiber-reinforced polymer composites under axial compression. Journal of Composites for Construction 13(3), 234-242. 
Bargmann, S., Kluseman, B., Markmann, J., Schnabel, J., Schneider, K., Soyarslan, C., Wilmers, J., 2018. Generation of 3d representative volume elements for heterogeneous materials: A review. Progress in Materials Science $96,322-384$.

Basu, S., Waas, A., Ambur, D., 2006. Compressive failure of fiber-composites under multi-axial loading. Journal of the Mechanics and Physics of Solids $54,611-634$.

Batra, R., Liu, D.-S., 1990. Adiabatic shear banding in dynamic plane strain compression of a viscoplastic material. International Journal of Plasticity $6,231-246$.

Batra, R., Love, B., 2004. Adiabatic shear bands in functionally graded materials. Journal of Thermal Stresses 27, 1101-1123.

Batra, R., Love, B., 2005. Mesoscale analysis of shear bands in high strain rate deformations of tungsten/nickel-iron composites. Journal of Thermal Stresses 28, 747-782.

Benaarbia, A., Rouse, J., Sun, W., 2018. A thermodynamically-based viscoelastic-viscoplastic model for the high temperature cyclic behaviour of 9-12\% Cr steels. International Journal of Plasticity 107, 100-121.

Beyerlein, I., Mayeur, J., Zheng, S., Mara, N., Wang, J., Misra, A., 2014. Emergence of stable interfaces under extreme plastic deformation. Proceedings of the National Academy of Sciences of the USA 111(12), 4386-4390. 
Cavalcante, M., Marques, S., Pindera, M., 2009. Transient thermomechanical analysis of a layered cylinder by the parametric finite-volume theory. Journal of Thermal Stresses 32, 112-134.

Chaboche, J., Kanoute, P., Ross, A., 2005. On the capabilities of mean field approaches for the description of plasticity in metal matrix composites. International Journal of Plasticity 21, 1409-1434.

Charalambakis, N., Baxevanis, T., 2004. Adiabatic shearing of nonhomogeneous thermoviscoplastic materials. International Journal of Plasticity 20, 899-914.

Charalambakis, N., Chatzigeorgiou, G., Chemisky, Y., Meraghni, F., 2018. Mathematical homogenization of inelastic dissipative materials: A survey and recent progress. Continuum Mechanics and Thermodynamics 30(1), $1-51$.

Chatzigeorgiou, G., Charalambakis, N., 2005. Instability analysis of nonhomogeneous materials under biaxial loading. International Journal of Plasticity 21, 1970-1999.

Chatzigeorgiou, G., Charalambakis, N., Chemisky, Y., Meraghni, F., 2018. Thermomechanical Behavior of Dissipative Composite Materials. ISTE Press - Elsevier, London.

Chatzigeorgiou, G., Charalambakis, N., Murat, F., 2009. Homogenization of a pressurized tube made of elastoplastic materials with discontinuous properties. International Journal of Solids and Structures 46, 3902-3913. 
Chatzigeorgiou, G., Kalpakides, V., Charalambakis, N., 2007. Biaxial loading of continuously graded thermoviscoplastic materials. Computational Mechanics 39, 335-355.

Chen, Q., Wang, G., Chen, X., 2018a. Three-dimensional parametric finitevolume homogenization of periodic materials with multi-scale structural applications. International Journal of Applied Mechanics in press, 30.

Chen, Q., Wang, G., Pindera, M., 2018b. Finite-volume homogenization and localization of nanoporous materials with cylindrical voids. part 1: Theory and validation. European Journal of Mechanics- A/Solids 70, 141-155.

Deng, X., Chawla, N., Chawla, K., Koopman, M., Chu, J., 2005. Mechanical behavior of multilayered nanoscale metal-ceramic composites. Advanced Engineering Materials 7(12), 1099-1108.

Dong, H., Cui, J., Nie, Y., Yang, Z., Wang, Z., 2018. High-order threescale computational method for heat-conduction problems of axisymmeric composite structures with multiple spatial scales. Advances in Engineering Software 121, 1-12.

Dudzinski, D., Molinari, A., 1991. Perturbation analysis of thermoviscoplastic instabilities in biaxial loading. International Journal of Solids and Structures 27(5), 601-628.

Edgren, F., Asp, L., Joffe, R., 2006. Failure of ncf composites subjected to combined compression and shear loading. Composites Science and Technology 66, 2865-2877. 
Fressengeas, C., Molinari, A., 1987. Instability and localization of plastic flow in shear at high strain rates. Journal of the Mechanics and Physics od Solids 35(2), 185-211.

Gotoh, M., Yanashita, M., Torii, N., 1993. Unexpected phenomena in multilayered copper subjected to plane strain compression. The Japan Society of Mechanical Engineers International Journal, Series A 36(2), 182-189.

Guinovart-Sanjuan, D., Vajravelou, K., Rodriguez-Ramos, R., GuinovartDiaz, R., Bravo-Castillero, J., Lebon, F., Sabina, F., 2018. Analysis of effective elastic properties for shell with complex geometrical shapes. Composite Structures 203, 278-285.

Jelf, P., Fleck, N., 1994. The failure of composite tubes due to combined compression and torsion. Journal of Materials Science 29, 3080-3084.

Katsaounis, T., Lee, M., Tzavaras, A., 2017. Localization of inelastic rate dependent shearing deformations. Journal of the Mechanics and Physics of Solids 98, 106-125.

Khatam, H., Pindera, M., 2010. Plasticity-triggered architectural effects in periodic multilayers with wavy microstructures. International Journal of Plasticity 26, 273-287.

Khedmati, M., Pedram, M., Rigo, P., 2014. The effects of geometrical imperfections on the ultimate strength of aluminium stiffened plates subject to combined uniaxial compression and lateral pressure. Ships and Offshore Structures 9(1), 88-109. 
Lopez-Realpozo, J., Rodriguez-Ramos, R., Guinovart-Diaz, R., BravoCastillero, J., Fernandez, L. P., Sabina, F., Maugin, G., 2008. Effective properties of non-linear elastic laminated composites with perfect and imperfect contact conditions. Mechanics of Advanced Materials and Structures 15(5), 375-385.

Love, B., Batra, R., 2006. Determination of effective thermomechanical parameters of a mixture of two elastothermoviscoplastic constituents. International Journal of Plasticity 22, 1026-1061.

Mander, J., Kim, J., Chen, S., 1993. Experimental performance and modeling study of a 30-year-old bridge with steel bearings. Transportation Research Records, 65-74.

Mara, N., Bhattacharyya, D., Hirth, J., Dickerson, P., 2010. Mechanism of shear banding in nanolayered composites. Applied Physics Letters 97(2), 021909 .

Michopoulos, J., Iliopoulos, A., , Steuban, J., Hermanson, J., 2018. Multiaxial and mulltiscale implications of dissipative behavior of composites. IOP Conf. Series: Material Science and Engineering 388, 1-22.

Nackenhorst, U., Kardas, D., Helmich, T., Lenz, C., Shan, W., 2011. Computational techniques for multiscale analysis of materials and interfaces. Lecture Notes in Applied and Computational Mechanics 57, 133-167.

Nie, G., Batra, R., 2010. Exact solutions and material tailoring for functionally graded hollow circular cylinders. Journal of Elasticity 99, 179-201. 
Nie, G., Zhong, Z., Batra, R., 2011. Material tailoring for functionally graded hollow cylinders and spheres. Composites Science and Technology 71, 666673.

Omri, A., Fennan, A., Sidoroff, F., Hihi, A., 2000. Elastic-plastic homogenization for layered composites. European Journal of Mechanics A/Solids 19, 585-601.

Ovid'ko, I., 2014. Metal-graphene nanocomposites with enhanced mechanical properties: A review. Review of Advanced Materials Science 38, 190-200.

Pindera, M., Khatam, H., Drago, S., Bansal, Y., 2009. Micromechanics of spatially uniform heterogeneous media: A critical review and emerging approaches. Composites Part B: Engineering 40, 349-378.

Rattez, H., Stefanou, I., Sulem, J., 2018a. The importance of thermo-hydromechanical couplings and microstructure to strain localization in 3D continua with application to seismic faults. Part I: Theory and linear stability analysis. Journal of Mechanics and Physics of Solids 115, 54-76.

Rattez, H., Stefanou, I., Sulem, J., Veveakis, M., Poulet, T., 2018b. The importance of Thermo-Hydro-Mechanical couplings and microstructure to strain localization in 3D continua with application to seismic faults. Part II: Numerical implementation and post-bifurcation analysis. Journal of Mechanics and Physics of Solids 115, 1-29.

Shen, Y.-L., Blada, C., Williams, J., Chawla, N., 2012. Cyclic indentation behavior of metal-ceramic nanolayered composites. Materials Science and Engineering A 557, 119-125. 
Terada, K., Kikuchi, N., 2001. A class of general algorithms for multi-scale analyses of heterogeneous media. Computer Methods in Applied Mechanics and Engineering 190, 5427-5464.

Tzavaras, A., 1987. Effect of thermal softening in shearing of strain-rate dependent materials. Archive for Rational Mechanics and Analysis 99(4), 349-374.

Zheng, S., Beyerlein, I., Carpenter, J., Kang, K., Wang, J., Han, W., Mara, N., 2013. High-strength and thermally stable bulk nanolayered composites due to twin-induced interfaces. Nature Communications, London 4, 1696.

Zhu, J., Wang, J., Ni, A., Guo, W., Li, X., Wu, Y., 2018. A multiparameter model for stiffness prediction of composite laminates with out-of-plane ply waviness. Composite Structures 185, 327-337. 


\section{Highlights}

We present the special solution of the two-dimensional problem of a continuously graded composite made of thermo-visco-rigid-plastic materials under combined biaxial quasi-static compression and tension (or compression) and shear, that preserves prescribed uniform normal strains.

In the context of non-linear instability, the results of linearized instability are examined, explained and corrected. Additionally, the destabilizing mechanism, the onset of instability and the critical time are defined and related to the lateral normal stress.

A semi-analytical homogenization scheme for a periodically-graded material is presented. Effective quantities such as shear strain-rate or dissipation-rate, are computed and compared with asymptotic homogenization results.

The homogenization result of the present paper can eventually be used in manufacturing processes of layered materials with functionally graded interfaces, for which prescribed uniform normal strains are desired under combined biaxial normal stress and shearing. 


\section{Highlights}

We present the special solution of the two-dimensional problem of a continuously graded composite made of thermo-visco-rigid-plastic materials under combined biaxial quasi-static compression and tension (or compression) and shear, that preserves prescribed uniform normal strains.

In the context of non-linear instability, the results of linearized instability are examined, explained and corrected. Additionally, the destabilizing mechanism, the onset of instability and the critical time are defined and related to the lateral normal stress.

A semi-analytical homogenization scheme for a periodically-graded material is presented. Effective quantities such as shear strain-rate or dissipation-rate, are computed and compared with asymptotic homogenization results.

The homogenization result of the present paper can eventually be used in manufacturing processes of layered materials with functionally graded interfaces, for which prescribed uniform normal strains are desired under combined biaxial normal stress and shearing. 